\title{
Cosmic rays across the star-forming galaxy sequence. II: Stability limits and the onset of cosmic ray-driven outflows
}

\author{
Roland M. Crocker, ${ }^{1 \star}$ Mark R. Krumholz, ${ }^{1}$ and Todd A. Thompson, ${ }^{2}$ \\ ${ }^{1}$ Research School of Astronomy and Astrophysics, Australian National University, Canberra 2611, A.C.T., Australia \\ ${ }^{2}$ Department of Astronomy and Center for Cosmology \& Astro-Particle Physics, The Ohio State University, Columbus, Ohio 43210, U.S.A
}

Accepted XXX. Received YYY; in original form ZZZ

\begin{abstract}
Cosmic rays (CRs) are a plausible mechanism for launching winds of cool material from the discs of star-forming galaxies. However, there is no consensus on what types of galaxies likely host CR-driven winds, or what role these winds might play in regulating galaxies' star formation rates. Using a detailed treatment of the transport and losses of hadronic CRs developed in the previous paper in this series, here we develop a semi-analytic model that allows us to assess the viability of using CRs to launch cool winds from galactic discs. In particular, we determine the critical CR fluxes and corresponding star formation rate surface densities - above which hydrostatic equilibrium within a given galaxy is precluded because CRs drive the gas off in a wind or otherwise render it unstable. We show that, for star-forming galaxies with lower gas surface densities typical of the Galaxy and local dwarfs, the locus of this CR stability curve patrols the high side of the observed distribution of galaxies in the Kennicutt-Schmidt parameter space of star formation rate versus gas surface density. However, hadronic losses render CRs unable to drive winds in galaxies with higher surface densities. Our results show that quiescent, low surface density galaxies like the Milky Way are poised on the cusp of instability, such that small changes to ISM parameters can lead to the launching of CR-driven outflows, and we suggest that, as a result, CR feedback sets an ultimate limit to the star formation efficiency of most modern galaxies.
\end{abstract}

Key words: hydrodynamics - instabilities - ISM: jets and outflows - radiative transfer - galaxies: ISM - cosmic rays

\section{INTRODUCTION}

This paper is the third in a series (Krumholz et al. 2020; Crocker et al. 2020a, hereafter Paper I) exploring the physics of relativistic cosmic ray (CR) transport, energy loss, and radiation in the interstellar media of star-forming galaxies, and, more importantly, the dynamical impact of CRs in such environments. In particular, our intention in this series is to investigate, in broad brush strokes, the potential importance of CRs as an agent of feedback in star-forming galaxies: What role, if any, do CRs have - as a function of environmental parameters - in establishing the remarkably low efficiency with which galaxies convert into stars the gas flowing out of the cosmic web and into their own interstellar media?

As discussed in Paper I and previously literature, CRs are a plausible agent of star formation feedback for a number of reasons: While this non-thermal particle population receives only a sub-dominant fraction, $\sim 10 \%$, of the to-

^ E-mail: rcrocker@fastmail.fm (RMC) tal kinetic energy liberated in supernova explosions, unlike the thermal gas (that receives most of the supernova energy), CRs lose energy to radiation very slowly. This means that, from their injection sites close to the midplanes of starforming galaxies, CRs tend to disperse well out into these galaxies' interstellar media ${ }^{1}$. Within the Milky Way disc, the CR energy density is near equipartition with the magnetic field and turbulent gas motions, implying CRs contribute significantly to establishing the vertical hydrostatic equilibrium of the gas (e.g., Boulares \& Cox 1990) and maintain, therefore, the conditions under which sustained, quiescent star-formation can proceed.

Moreover, given their soft effective equation of $\mathrm{state}^{2}$, CRs come to increasingly dominate the total energy density of a co-mingled astrophysical fluid of thermal and non-

1 In fact, in many cases, including for the Milky Way, they may escape the galactic disc completely.

2 That follows from the fact that the energetically dominant cosmic ray population is relativistic, i.e., adiabatic index $\gamma_{c} \rightarrow 4 / 3$. 
thermal particles that is suffering adiabatic losses under expansion in an outflow. Thus CRs can help sustain galactic winds by providing a distributed heating source via their non-adiabatic energy losses which, in this situation, are mostly mediated by the streaming instability (e.g., Everett et al. 2008; Zweibel 2017; Ruszkowski et al. 2017). Despite, however, the early recognition of their potential importance in driving winds (Ipavich 1975; Breitschwerdt et al. 1991; Zirakashvili et al. 1996; Ptuskin et al. 1997), the possibility that CRs might generically be an important source of feedback in galaxy formation has only recently begun to receive much sustained attention, in either phenomenological (e.g., Zirakashvili \& Völk 2006; Everett et al. 2008; Samui et al. 2010; Crocker et al. 2011; Crocker 2012; Lacki et al. 2011; Hanasz et al. 2013; Yoast-Hull et al. 2016), or numerical models (e.g., Jubelgas et al. 2008; Wadepuhl \& Springel 2011; Uhlig et al. 2012; Booth et al. 2013; Salem \& Bryan 2014; Salem et al. 2016; Pakmor et al. 2016; Simpson et al. 2016; Recchia et al. 2016, 2017; Ruszkowski et al. 2017; Pfrommer et al. 2017; Chan et al. 2019; Buck et al. 2019). Even so, there remains significant disagreement in the literature about where and when CRs might be important: some authors conclude they are capable of driving galactic winds only off the most rapidly star-forming galaxies (e.g., Socrates et al. 2008), while others find they drive winds only in dwarfs, (e.g., Jubelgas et al. 2008; Uhlig et al. 2012), and yet others that they do not drive winds by themselves at all, but can reheat and energise winds launched by other processes (e.g. Ruszkowski et al. 2017).

Thus, a first-principles effort to understand where and when CRs might be important, taking into account all the available observational constraints, seems warranted, and this is the primary goal of this and our previous paper. Having explored the theory and observational consequences of $\mathrm{CR}$ transport in the largely neutral gas phase from which star form (Krumholz et al. 2020), here and in our previous paper (Paper I), we seek to cut a broad swathe across the parameter space of star-forming galaxies, and determine where within this parameter space CRs might be important agents of feedback. We break this task down into two parts. Paper I addresses the question: What fraction of the total ISM pressure is typically supplied by CRs as a function of galaxy parameters? In other words: How important to the overall gas dynamics in typical star-forming galaxies can CRs be? In this paper we use the mathematical set-up of our previous papers to address a rather specific, follow-up question: What is the critical flux of cosmic rays above which a hydrostatic equilibrium within a given column of gas is precluded? In other words: At what point do cosmic rays - accelerated as a result of the star formation process itself - start to drive outflows in galaxies? We emphasise that we are not addressing the question of whether CRs can re-accelerate or re-heat winds that have been launched by other mechanisms, a question addressed by a number of previous authors as discussed above. Instead, we seek to determine under what conditions it becomes inevitable that the CRs themselves begin to lift neutral interstellar gas out of galactic discs, certainly rendering the neutral gas atmosphere unstable, and potentially giving rise to a cool galactic wind.

The remainder of this paper is structured as follows: in Section 2 we briefly recap the mathematical setup of the problem and, in particular, write down the ordinary differen- tial equation (ODE) system that describes a self-gravitating gaseous disc that maintains a quasi-hydrostatic equilibrium while subject to a flux of CRs injected at its midplane; in Section 3 we present, describe, and evaluate the numerical solutions of our ODEs; in Section 4 we consider the astrophysical implications of our findings for CR feedback on the dense, star-forming gas phase of spiral galaxies; we further discuss our results and summarise in Section 5 .

\section{SETUP}

\subsection{Physical Model: Recapitulation}

We provide a detailed description of the physical system we model in the companion paper (Paper I). In brief, our model is similar to one previously invoked by us in studies of radiation pressure feedback (Krumholz \& Thompson 2012, 2013; Crocker et al. 2018a,b; Wibking, Thompson, \& Krumholz 2018): an idealised 1D representation of a portion of a galactic disc with total gas mass per unit area $\Sigma_{\text {gas }}$ and gas fraction $f_{\text {gas }}$, supported by a combination of turbulent motions with velocity dispersion $\sigma$, magnetic fields, and CR pressure, and confined by gravity. CRs (or radiation) are injected into this medium at the midplane with flux $F_{c, 0}$. In the radiation context we have previously shown that, when the injected radiation flux exceeds a critical value, the system is destabilised and equilibrium becomes impossible. Numerical simulations confirm that radiation-driven winds are possible only in those systems for which equilibria do not exist. Here we are interested to determine whether a similar critical flux exists for CRs, since, if it does, that would suggest the circumstances under which it is possible for CRs to launch outflows of material out of galactic discs.

\subsubsection{Equations for transport and momentum balance}

In Paper I we provide a detailed derivation of a pair of coupled ordinary differential equations (ODEs) that describe hydrostatic equilibrium and transport of CRs with losses. We present only a sketch of this development here for convenience, and refer readers to Paper I for the full derivation. We treat CRs in the relativistic, fluid dynamical limit whereby they behave as a fluid of adiabatic index $\gamma_{c}=4 / 3$. Our ODEs express how the CR pressure and the gas column change as a function of our single variable, $z$, the height above the midplane. CRs are assumed to be injected by supernova explosions occurring solely in a thin layer near $z=0$; in the context of establishing a stability limit, this assumption turns out to be conservative (even though it is not realistic for most galaxies). We show in Paper I that the system can be described in terms of four dimensionless functions, $s(\xi), r(\xi)=d s / d \xi, p_{c}(\xi)$, and $\mathcal{F}_{c}(\xi)$, which represent the dimensionless gas column, gas density, CR pressure, and CR flux as a function of dimensionless height $\xi$. These functions are prescribed by two equations. The first is the dimensionless CR transport equation,

$$
\frac{\tau_{\text {stream }}}{\beta_{S}} \frac{d \mathcal{F}_{c}}{d \xi}=-\tau_{\text {abs }} r p_{c}+\tau_{\text {stream }} \frac{d p_{c}}{d \xi},
$$

where $\tau_{\text {stream }}, \tau_{\text {abs }}$, and $\beta_{S}$ are all defined below and

$$
\mathcal{F}_{c}=-\frac{\beta_{s}}{\tau_{\text {stream }}} r^{-q} \frac{d p_{c}}{d \xi}
$$


is the dimensionless CR flux expressed in the standard diffusion approximation (Ginzburg \& Syrovatskii 1964) ${ }^{3}$, in which $q$ specifies the running of the diffusion coefficient with density (i.e., the diffusion coefficient is proportional to $\rho^{-q}$ ). The term on the LHS of equation 1 represents the gradient of the CR flux, while the two terms on the RHS represent, respectively, collisional and streaming losses of the $\mathrm{CRs}^{4}$. The coupled ODE expressing hydrostatic balance is

$$
\frac{d p_{c}}{d \xi}+\phi_{\mathrm{B}} \frac{d r}{d \xi}=-\left(1-f_{\mathrm{gas}}\right) r-f_{\mathrm{gas}} s r .
$$

The terms in equation 3 are, from left to right, the pressure gradient due to CRs, the pressure gradient due to combined turbulence (treated as isotropic) plus magnetic support ${ }^{5}$, the gravitational acceleration due to stellar gravity, and the acceleration due to gas self-gravity.

The dimensionless variables are related to the physical quantities as follows. The dimensionless height is the physical height measured in units of the turbulent scale height:

$$
\xi \equiv \frac{z}{z_{*}}
$$

where

$$
z_{*} \equiv \frac{\sigma^{2}}{g_{*}},
$$

(in which the turbulent velocity dispersion of the gas $\sigma$ is assumed constant) and

$$
g_{*}=2 \pi G \frac{\Sigma_{\mathrm{gas}}}{f_{\mathrm{gas}}} .
$$

Similarly $s(\xi)$ is the (dimensionless) fraction of the total half column contributed by gas in the height range from 0 to $\xi z_{*}$, and $p_{c}(\xi)$ is the dimensionless CR pressure obtained by normalizing the dimensional CR pressure to the characteristic midplane pressure $P_{*}$ (with related energy density $\left.u_{*}=(3 / 2) P_{*}\right)$ given by

$$
P_{*}=g_{*} \rho_{*} z_{*}=\rho_{*} \sigma^{2}=\frac{\pi G}{f_{\mathrm{gas}}} \Sigma_{\mathrm{gas}}^{2} \simeq 0.57 \frac{\Sigma_{\mathrm{gas}, 1}^{2}}{f_{\mathrm{gas}}} \mathrm{eV} \mathrm{cm}^{-3},
$$

where we have defined $\Sigma_{\text {gas, } 1}=\Sigma_{\text {gas }} /\left(10 M_{\odot} \mathrm{pc}^{-2}\right)$ and

$$
\rho_{*} \equiv \frac{\Sigma_{\text {gas }}}{2 z_{*}}
$$

is the characteristic matter density. The local density as a function of height is $\rho_{*}(d s / d \xi)$.

Other parameters appearing in the coupled ODEs are as follows: The coefficients $\tau_{\text {abs }}$ and $\tau_{\text {stream }}$ appearing on the RHS of equation 1 are, respectively, the optical depths of the gas column to CR absorption and scattering (see equation 9 and equation 10 below). In equation $3, \phi_{B}$ on the LHS lies in the range 0 to 2 and specifies the importance of magnetic effects in modifying the pressure due solely to gas turbulence

\footnotetext{
3 Note that we can describe the process in terms of diffusion even if the microphysical transport process is predominantly streaming, as long as we are averaging over scales comparable to or larger than the coherence length of the magnetic field - see Krumholz et al. (2020) for further discussion.

4 Note that here we assume that second-order Fermi reacceleration is negligibly small or actually zero; cf. Zweibel (2017).

5 Note that we thus implicitly assume negligible thermal pressure.
}

(with values $>1$ indicating magnetic pressure support and values $<1$ indicating confinement by magnetic tension). We adopt $q=1 / 4$ and $\phi_{B}=73 / 72$ as fiducial values, but our results are only weakly sensitive to these choices - for further discussion see Paper I.

The cosmic ray optical depth parameters are given by

$$
\begin{aligned}
\tau_{\text {stream }} & =\frac{\beta_{s}}{K_{*} \beta} \\
\tau_{\mathrm{abs}} & =\frac{1}{K_{*} \beta} \tau_{\mathrm{pp}} .
\end{aligned}
$$

where $\beta_{s} \equiv v_{s} / c$ denotes the dimensionless CR streaming speed, $\beta \equiv \sigma / c$ is the dimensionless ISM velocity dispersion, and $K_{*}$ is the dimensionless midplane CR diffusion coefficient expressed in units of the effective diffusion coefficient for convective transport:

$$
\kappa_{*}=K_{*} \kappa_{\mathrm{conv}},
$$

where

$$
\begin{aligned}
\kappa_{\mathrm{conv}} & =\frac{z_{*} \sigma}{3}=\frac{\sigma^{3} f_{\mathrm{gas}}}{6 \pi G \Sigma_{\mathrm{gas}}} \\
& \simeq 3.8 \times 10^{26} \mathrm{~cm}^{2} \mathrm{~s}^{-1} \sigma_{1}^{3} \mathrm{f}_{\mathrm{gas}} \Sigma_{\text {gas }, 1}^{-1},
\end{aligned}
$$

and we have defined $\sigma_{1}=\sigma / 10 \mathrm{~km} \mathrm{~s}^{-1}$. Note that, as convection sets a lower limit to the rate of diffusion, $K_{*} \geqslant 1$. We apply this limit to all of the CR transport models we

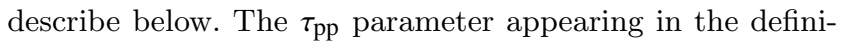
tion of $\tau_{\text {abs }}$ is the optical depth for "absorption" of cosmic rays via the hadronic collisions they experience in the limit of rectilinear propagation at speed $c$ from the midplane to infinity through (half of the total) gas column $\Sigma_{\text {gas }}$ :

$$
\tau_{\mathrm{pp}}=\frac{\Sigma_{\mathrm{gas}}}{2 \Sigma_{\mathrm{pp}}}
$$

where

$\Sigma_{\mathrm{pp}} \equiv \frac{\mu_{p} m_{p}}{3 \eta_{\mathrm{pp}} \sigma_{\mathrm{pp}}} \simeq \frac{33 \mathrm{~g} \mathrm{~cm}^{-2}}{\left(\eta_{\mathrm{pp}} / 0.5\right)\left(\sigma_{\mathrm{pp}} / 40 \mathrm{mbarn}\right)} \simeq 1.6 \times 10^{5} M_{\odot} / \mathrm{pc}^{2}$

is the grammage required to decrease the CR flux by one $e$ folding; here $m_{p}$ is the proton mass, $\mu_{p} \simeq 1.17$ is the number of protons per nucleon for gas that is $90 \% \mathrm{H}, 10 \% \mathrm{He}$ by number, $\eta_{\mathrm{pp}}$ and $\sigma_{\mathrm{pp}}$ are the inelasticity and total crosssection for hadronic collisions experienced by relativistic CR protons.

The system formed by equation 1 and equation 3 is fourth-order, and thus requires four boundary conditions. Two of these apply to the density, and are

$$
\begin{aligned}
s(0) & =0 \\
\lim _{\xi \rightarrow \infty} s(\xi) & =1,
\end{aligned}
$$

which amount to asserting that the gas half column is zero at the midplane, and that $\lim _{z \rightarrow \infty} \Sigma_{\text {gas, } 1 / 2}(z)=1 / 2 \Sigma_{\text {gas }}$. The remaining two apply to the CR pressure and flux, and are

$$
\frac{\tau_{\text {stream }}}{\beta_{S}} \mathcal{F}_{c}(0)=\frac{1}{K_{*} \beta} \frac{F_{c, 0}}{F_{*}} \equiv f_{\text {Edd }},
$$

at $\xi=0$ and

$$
\lim _{\xi \rightarrow \infty} \mathcal{F}_{c}=\lim _{\xi \rightarrow \infty} 4 \beta_{s} p_{c} .
$$

The first of these, equation 17 , is set by the CR flux $F_{c, 0}$ 
entering the gas column; here $F_{*}=c P_{*}$ is the scale flux for our non-dimensional system, and $f_{\text {Edd }}$ is the Eddington ratio, which gives the ratio of the momentum flux carried by the CRs to that imparted by gravity. The second, equation 18, asserts that the CR flux approach the value for free-streaming as $\xi \rightarrow \infty$. Again, we refer readers to Paper I for a full derivation of these conditions.

\subsection{CR transport models}

To complete the specification of the system, we require expressions for $K_{*}$ and $\beta_{s}$, the normalised CR diffusion coefficient and streaming speed. These depend on the microphysics of CR confinement, and here we consider the same three models for this process as in Paper I. These are:

\subsubsection{Streaming (fiducial case)}

We are interested in the feedback effects of CRs on the (predominantly) neutral ISM, which at the midplane of a galactic disc constitutes $\sim 50 \%$ of the volume (Dekel et al. 2019), and close to $100 \%$ in the densest starbursts (Krumholz et al. 2020), and the vast majority of the mass. Thus our fiducial case is for CR transport through such a medium. As discussed in Paper I and shown in Krumholz et al. (2020), in such a medium strong ion-neutral damping prevents interstellar turbulence from cascading down to the small scales of CR gyroradii, which are the only scales that efficiently scatter CRs. Thus the only disturbances in the magnetic field with which CRs interact are those they themselves generate via the streaming instability. Thus CRs stream along field lines, but for the relatively low (but still relativistic) $\mathrm{CR}$ energies that dominate the CR energy budget, streaming instability limits the streaming speed to the ion Alfvén velocity of the medium,

$$
v_{A, i}=\frac{\sigma}{\sqrt{2 \chi} M_{A}},
$$

where $M_{A}$ is the Alfvén Mach number of the Alfvénic turbulence modes in the ISM and $\chi$ is the ionisation fraction by mass. For a dynamo-generated field $M_{A} \approx 1-2$ (Federrath et al. 2014; Federrath 2016, Paper I), and astrochemical models show that the ionisation fraction $\chi$ ranges from $\sim 10^{-2}$ in Milky Way-like galaxies with relatively diffuse neutral media (Wolfire et al. 2003) to $\sim 10^{-4}$ in dense starbursts Krumholz et al. (2020). On larger scales, the CR diffusion coefficient is therefore set by the combination of streaming at this speed along the field lines, and the random walk of the field lines themselves in the turbulence. For this model, we show in Paper I that

$$
\begin{aligned}
K_{*} & =\frac{1}{\sqrt{2 \chi} M_{A}} \\
\beta_{S} & =\frac{\beta}{\sqrt{2 \chi} M_{A}} \\
\tau_{\text {stream }} & =M_{A}^{3} \\
\tau_{\text {abs }} & =\frac{\sqrt{2 \chi} M_{A}^{4}}{\beta} \tau_{\mathrm{pp}},
\end{aligned}
$$

and we use $q=1 / 4$ as our fiducial choice as introduced above $^{6}$. For a given choice of $M_{A}$ and $\chi$, and a galactic disc of specified $\Sigma_{\text {gas }}$ and $\sigma$ (which set $\tau_{\text {pp }}$ and $\beta$, respectively), these expressions complete the specification of the system.

\subsubsection{Scattering}

Our second model is based on the premise that, although we are interested in feedback on the neutral ISM, ionised gas nevertheless fills $\sim 50 \%$ of the midplane volume in most galaxies (Cox \& Smith 1974; Dekel et al. 2019), with the fraction rising as one goes away from the midplane, and thus CR transport might take place predominantly in the ionised phase of the ISM. Indeed, in situ observations suggest that such is the case for the local CR population seen at Earth (e.g., Ghosh \& Ptuskin 1983; Jones et al. 2001). In this case CRs may still interact predominantly with their own selfgenerated turbulence, in which case we return to a situation much like the streaming model, except with $\chi=1$. The more interesting possibility, therefore, is that, although CRs do stream at speed $v_{S}=v_{A, i}=\sigma / \sqrt{2} M_{A}$, they also scatter off turbulence that is part of the large-scale turbulent cascade in the ISM, and that this scattering is what sets the diffusion coefficient. In this case, we show in Paper I that transport coefficients are given by

$$
\begin{aligned}
K_{*} & =\frac{1}{\beta}\left(\frac{G}{2 f_{\text {gas }}}\right)^{p / 2}\left(\frac{E_{\mathrm{CR}} M_{A}}{e \sigma^{2}}\right)^{p} \\
\beta_{s} & =\frac{\beta}{\sqrt{2} M_{A}} \\
\tau_{\text {stream }} & =\frac{3 \beta}{\sqrt{2} M_{A}^{2}}\left(\frac{E_{\mathrm{CR}}}{e \sigma^{2}} \sqrt{\frac{G}{2 f_{\mathrm{gas}}}}\right)^{-p} \\
\tau_{\mathrm{abs}} & =\frac{3 \tau_{\mathrm{pp}}}{M_{A}}\left(\frac{E_{\mathrm{CR}}}{e \sigma^{2}} \sqrt{\frac{G}{2 f_{\mathrm{gas}}}}\right)^{-p} .
\end{aligned}
$$

Here $E_{\mathrm{CR}}$ is the CR energy (we adopt $E_{\mathrm{CR}}=1 \mathrm{GeV} \equiv E_{\mathrm{CR}, 0}$ as a fiducial choice), $e$ is the elementary charge, and $p$ is the index of the turbulent power spectrum $-p=1 / 3$ (corresponding to $q=1 / 6$ ) for a Kolmogorov spectrum, and $p=1 / 2$ (corresponding to $q=1 / 4$, i.e., the fiducial value) for a Kraichnan spectrum, though which value of $p$ we choose makes little difference to the qualitative results. As with the streaming model described above, for a particular choice of $E_{\mathrm{CR}, 0}, p$, and $M_{A}$, the above expressions allow us to compute the transport coefficients $\beta_{s}, \tau_{\text {stream }}$, and $\tau_{\text {abs }}$ for any choice of galactic disc parameters $\Sigma_{\text {gas }}$ and $\sigma$. Compared to the streaming model, the scattering model generally predicts smaller streaming optical depths in all galaxies, and comparable diffusion rates and absorption optical depths in Milky Way-like galaxies. The models differ mainly in their predictions for denser and more rapidly star-forming galaxies, where the scattering model predicts slower transport and greater absorption optical depths than in the Milky Way

6 This corresponds to the physical limit where the turbulent velocity dispersion is density independent, there is a local turbulent dynamo acting, and the ionization fraction becomes independent of the local gas density; while this latter is unlikely to hold strictly, as we have previously shown (Paper I and Krumholz et al. 2020), our results are not strongly dependent on $q$ so long as $0<q<1$. 
(due to stronger turbulence), while the streaming model predicts the opposite (due to the lower ionisation fraction in denser galaxies allowing faster $\mathrm{CR}$ streaming). We provide a more detailed comparison in Paper I.

\subsubsection{Constant diffusion coefficient}

The third model we consider is a purely empirical one: The empirically-determined diffusion coefficient for $\sim \mathrm{GeV}$ CRs in the Milky Way is close to $\kappa_{*, \mathrm{MW}} \equiv 10^{28} \mathrm{~cm}^{2} \mathrm{~s}^{-1}$ (e.g., Ptuskin et al. 2006) and, in this model, we simply assume $\kappa$ in all galaxies is given by this value. We thus assume that CRs stream through a fully ionised medium, as in the scattering case, but we take the dimensionless diffusion coefficient to be $K_{*}=\kappa_{*} \mathrm{MW} / \kappa_{\text {conv }}$. The corresponding expressions for the dimensionless numbers entering the equilibrium equations are

$$
\begin{aligned}
K_{*} & =\frac{6 \pi G \Sigma_{\mathrm{gas}} \kappa_{*, \mathrm{MW}}}{f_{\mathrm{gas}} \sigma^{3}} \\
\beta_{S} & =\frac{\beta}{\sqrt{2} M_{A}} \\
\tau_{\text {stream }} & =\frac{1}{\sqrt{2} M_{A}}\left(\frac{\kappa_{\mathrm{conv}}}{\kappa_{*, \mathrm{MW}}}\right) \\
\tau_{\mathrm{abs}} & =\frac{\tau_{\mathrm{pp}}}{\beta}\left(\frac{\kappa_{\mathrm{conv}}}{\kappa_{*, \mathrm{MW}}}\right) .
\end{aligned}
$$

Since we can write down the convective diffusion coefficient $\kappa_{\text {conv }}$ as a function of $\Sigma_{\text {gas }}$ and $\sigma$ (c.f. equation 12), this again represents a complete specification of the system.

\section{THE COSMIC RAY EDDINGTON LIMIT}

With this review of our dimensionless ODE system, and having dealt with the microphysics of CR transport, we are now in a position to address the basic question posed in this paper: under what conditions does it become impossible for a galactic disc forced by CRs from below to remain hydrostatic? To answer this question, we first describe a numerical method to identify this limit in the space of the dimensionless variables that characterise our system (Section 3.1), we use this method to obtain critical stability curves in this space (Section 3.2), and then we translate from the space of dimensionless variables to the space of observable galaxy properties (Section 3.3).

\subsection{Numerical method}

We must solve equation 1 and equation 3 numerically. Because the boundary conditions for the system, equation 15 - equation 18, are specified at different locations, the system forms a boundary value problem, which we solve using a shooting algorithm as follows: we have $s(0)=0$ from equation 15 , and we start with an initial guess for the mid-plane density $r(0)=s^{\prime}(0)$ and pressure $p_{c}(0)$. These choices together with equation 17 allow us to compute the midplane CR pressure gradient $p_{c}^{\prime}(0)$, so that we now have a set of four initial values at $s=0$ and can integrate outwards until $s(\xi)$ and $p_{c}(\xi)$ approach constant values at large $\xi$. In general our guess will not satisfy equation 16 , i.e., $s(\xi)$ will go to a value other than unity as $\xi \rightarrow \infty$. We therefore iteratively adjust
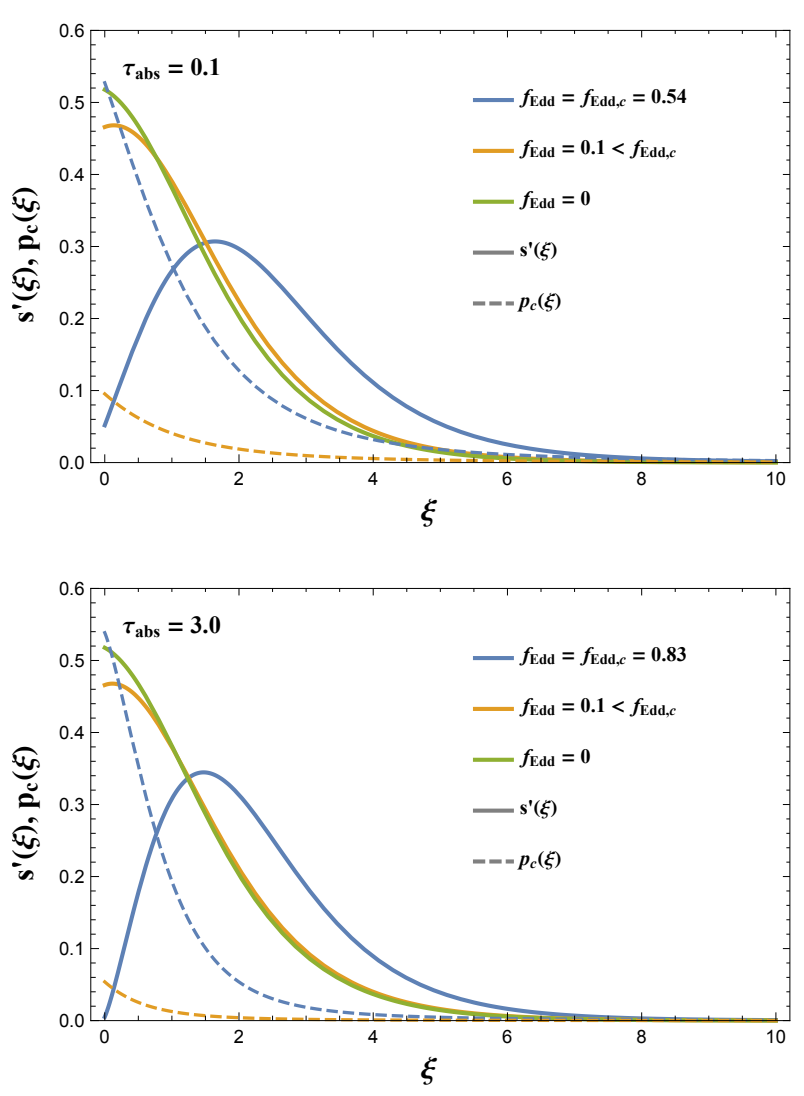

Figure 1. Profiles of (dimensionless) volumetric density $r(\xi)=$ $s^{\prime}(\xi)$ (solid) and (dimensionless) CR pressure $p_{c}(\xi)$ (dashed) for various representative cases as indicated in the legend. The upper panel is for $\tau_{\mathrm{abs}}=0.1$, the lower is for $\tau_{\mathrm{abs}}=3.0$; otherwise, parameters common between the panels are $q=1 / 4, \tau_{\text {stream }}=1, f_{\text {gas }}=$ $0.9, \phi_{\mathrm{B}}=73 / 72$. In both panels, the blue curves are evaluated for the critical $f_{\text {Edd }}$ case and the yellow curves are for a sub-critical $f_{\text {Edd }}$ value; the solid green curve is the density profile of a gas column supported purely by turbulence (with $\phi_{\mathrm{B}}=73 / 72$ ). Note that because $\sigma$ is constant, $s^{\prime}(\xi)$ is equivalent to the dimensionless turbulent pressure, and thus the ratio of solid and dashed curves of the same colour is also the ratio of turbulent to CR pressure.

$s^{\prime}(0)$ while holding $p_{c}(0)$ fixed, until equation 16 is satisfied. In general, however, this choice will not obey equation 18 , i.e., the CR flux will not go to the correct value as $\xi \rightarrow \infty$. We therefore now iteratively adjust our guess for $p_{c}(0)$. We continue to iterate between our guesses for $s^{\prime}(0)$ and $p_{c}(0)$ until the system converges and all boundary conditions are satisfied, or until convergence fails (see below).

A crucial feature of solutions to this system is that, as $f_{\text {Edd }}$ increases at fixed $\tau_{\text {abs }}$ and $\tau_{\text {stream }}$, the dimensionless midplane density $s^{\prime}(0)$ decreases monotonically, approaching zero at a finite value of $f_{\text {Edd }}$. We illustrate this behaviour for two example cases in Figure 1. We refer to the value of $f_{\mathrm{Edd}}$ for which this occurs as the critical Eddingtion ratio, $f_{\mathrm{Edd}, \mathrm{c}}$. No solutions exists for $f_{\text {Edd }}>f_{\text {Edd,c }}$, and thus $f_{\text {Edd,c }}$ represents the largest Eddington ratio for which it is possible for a gas column through which CRs are forced to remain in equilibrium. Larger values of $f_{\text {Edd }}$ necessarily render the system unstable. Mathematically, this manifests in that we 
are unable to find values of $s^{\prime}(0)$ and $p_{c}^{\prime}(0)$ such that, when we integrate equation 1 and equation 3 , the resulting solution satisfies the boundary conditions equation 16 and equation 18 as $\xi \rightarrow \infty$. The shooting method fails to converge.

We determine the value of the critical Eddington ratio $f_{\text {Edd,c }}\left(\tau_{\text {abs }}, \tau_{\text {stream }}\right)$ as a function of $\tau_{\text {abs }}$ and $\tau_{\text {stream as follows. }}$ We start with a small value of $f_{\text {Edd }}$, for which a solution is guaranteed to exist because in the limit $f_{\text {Edd }} \rightarrow 0$, equation 1 and equation 3 are completely decoupled; the former just reduces to the equation for an isothermal atmosphere, and the latter to a nonlinear diffusion equation with losses, the analytic solution for which is given by Krumholz et al. (2020). We use the shooting procedure described above to obtain the numerical solution for this small value of $f_{\text {Edd }}$. We then progressively increase $f_{\text {Edd }}$ and solve again, using the solution for the previous value as a starting guess. Eventually we reach a value of $f_{\text {Edd }}$ for which the shooting method fails to converge, and no solution exists. Once we find this value, we iteratively decrease and increase $f_{\text {Edd }}$ in order to narrow down the value $f_{\text {Edd,c }}$ for which a solution ceases to exist. We iterate in this manner until we have determined $f_{\text {Edd,c }}$ for a given $\tau_{\text {abs }}$ and $\tau_{\text {stream }}$ to some desired tolerance. Figure 1 confirms that the value of $f_{\text {Edd,c }}$ we obtain by this procedure is indeed such that $s^{\prime}(0)$ is close to zero, although in practice how close we are able to push $s^{\prime}(0)$ to zero depends on the tolerances we use in our iterative solver - in the vicinity of $f_{\mathrm{Edd}, \mathrm{c}}$, the value of $s^{\prime}(0)$ becomes exquisitely sensitive to $f_{\text {Edd }}$. This is visible in the upper panel of Figure 1 , where our solution for $f_{\text {Edd }} \approx f_{\text {Edd,c }}$ has $s^{\prime}(0) \approx 0.05$, but if we increase $f_{\text {Edd }}$ by even $1 \%$, then solutions cease to exist entirely.

\subsection{Critical curves}

We show sample values of $f_{\text {Edd,c }}$ determined via the procedure described in Section 3.1 in Figure 2; the top panel shows $f_{\text {Edd,c }}$ as a function of $\tau_{\text {abs }}$ for fixed $\tau_{\text {stream }}$ at several values of $f_{\text {gas }}$, while the bottom panel shows $f_{\text {Edd,c }}\left(\tau_{\text {abs }}\right)$ for fixed $f_{\text {gas }}$ at several values of $\tau_{\text {stream. }}$. Qualitatively, the behaviour of the solution with respect to $\tau_{\mathrm{abs}}$ is that, at small $\tau_{\mathrm{abs}}, f_{\mathrm{Edd}, \mathrm{c}}$ approaches a fixed, $O(1)$ value. At large $\tau_{\mathrm{abs}}$, we find that $f_{\text {Edd,c }}$ begins to scale $\underset{\sim}{\propto} \tau_{\text {abs }}$. We also find that, at low $\tau_{\text {abs }}$, we have a rough scaling $f_{\text {Edd, }} \propto \tau_{\text {stream scaling (cf. lower panel }}$ of Figure 2). Finally, we find that increasing $f_{\text {gas }}$ renders the column less stable for other parameters held fixed; this is as expected given that gas self-gravity must vanish in the midplane.

We can understand the observed scalings of $f_{\text {Edd,c }}$ with $f_{\text {gas }}, \tau_{\text {abs }}$, and $\tau_{\text {stream }}$ via some straightforward analytic considerations. Of these, $f_{\text {gas }}$ is the simplest. We note that, in the limit $f_{\text {Edd }} \ll 1$, the equation of hydrostatic balance (equation 3 ) has the usual solutions $s^{\prime}(\xi)=e^{-\xi / \phi_{B}}$ for $f_{\text {gas }}=0$ and $s^{\prime}(\xi)=\operatorname{sech}^{2}\left(\xi / 2 \phi_{B}\right)$ for $f_{\text {gas }}=1$; at $\xi=0$, these solutions have $s^{\prime}=1 / \phi_{B}$ and $1 / 2 \phi_{B}$, respectively, so the density at the midplane is twice as high with $f_{\text {gas }}=0$ as with $f_{\text {gas }}=1$. In between these limits, the midplane density scales as approximately $1 /\left[\phi_{B}\left(1+f_{\text {gas }}\right)\right]$. Since the critical $f_{\text {Edd }}$ corresponds to the point where $s^{\prime}(0) \rightarrow 0$, we expect that configurations starting with a larger value of $s^{\prime}(0)$ at low $f_{\text {Edd }}$ should have higher $f_{\text {Edd,c }}$, as $\left.f_{\text {Edd, }} \propto s^{\prime}(0)\right|_{f_{\text {Edd }} \ll 1}$. This suggests a scaling $f_{\text {Edd,c }} \stackrel{\propto}{\sim} 1 /\left[\phi_{B}\left(1+f_{\text {gas }}\right)\right]$, which is consistent with our numerical results.
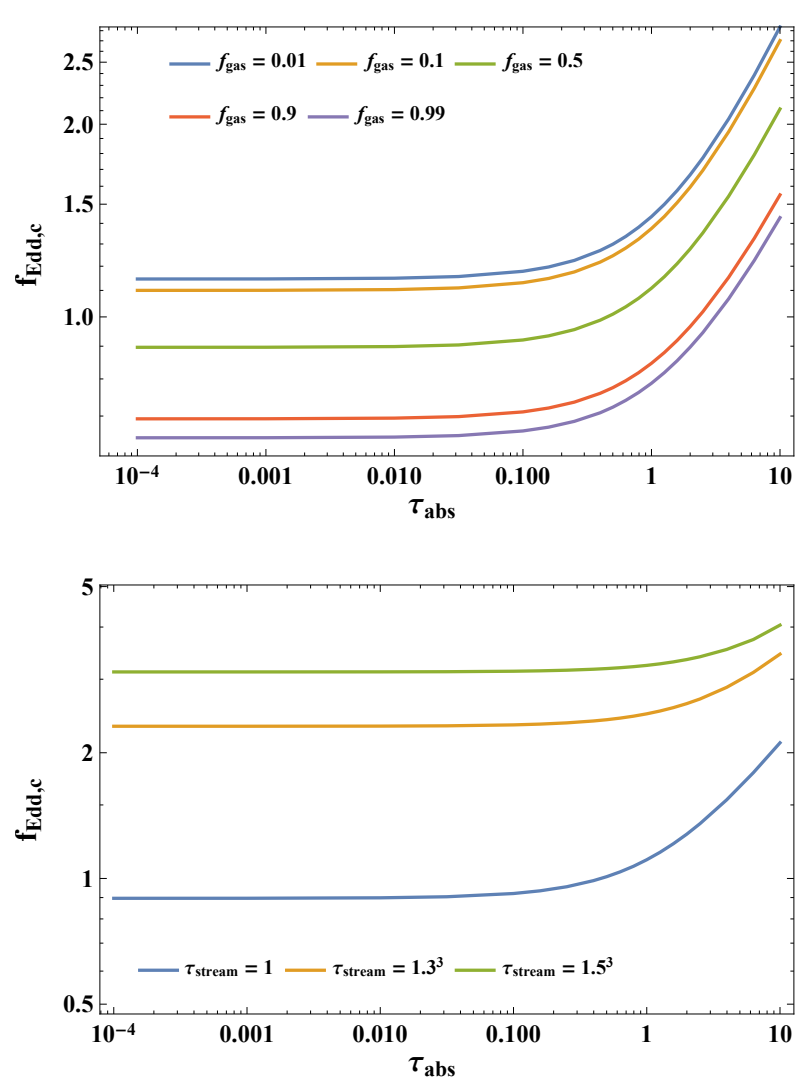

Figure 2. CR Eddington limit $f_{\mathrm{Edd}, \mathrm{c}}$ as a function of effective optical depth $\tau_{\text {abs }}$. Upper panel: $f_{\text {Edd,c }}$ versus $\tau_{\text {abs }}$ at fixed $\tau_{\text {stream }}=$ 1 and for a range of gas fractions $f_{\text {gas }}$ as indicated in the legend; a higher $f_{\text {gas }}$ renders the column (somewhat) less stable (i.e., reduces $f_{\text {Edd,c }}$ ), while a higher $\tau_{\text {abs }}$ renders the column more stable once we are in the optically thick regime (because hadronic collisions reduce the steady state CR pressure). Lower panel: $f_{\text {Edd,c }}$ as a function of $\tau_{\text {abs }}$ for fixed $f_{\text {gas }}=0.5$ and a range of $\tau_{\text {stream }}$ values as indicated in the legend; a higher $\tau_{\text {stream }}$ renders the column more stable (again because losses - in this instance due to the streaming instability - reduce the steady state CR pressure).

The scalings for $\tau_{\mathrm{abs}}$ and $\tau_{\text {stream }}$ require only slightly more consideration. We expect our system to approach the critical limit when the midplane CR pressure, $P_{c}$, becomes significant in comparison to the pressure required to keep the column in hydrostatic equilibrium, $P_{*}$. In steady state, the midplane CR pressure (or energy density, which differs just by a factor of 3 ), in turn, will be set by the product of the CR energy injection rate - set by star formation - and a dwell time $t_{c}$ for CRs injected into the galaxy. Thus we have

$$
\left(\frac{P_{c}}{P_{*}}\right)_{z=0} \sim \frac{\left(F_{c, 0} / z_{*}\right) t_{c}}{P_{*}}=\frac{\sigma t_{c}}{z_{*}} K_{*} f_{\mathrm{Edd}}
$$

where $F_{c, 0}$ is the energy injected per unit area, and we write the energy injected per unit volume as $F_{c, 0} / z_{*}$ under the assumption that the CRs are distributed over a height of order $z_{*}$. In the second step, we made use of equation 17 to rewrite $F_{c, 0}$ in terms of the Eddington ratio.

The dwell time for a CR will be set by the minimum of 
the time required for it to be lost to a collision, $t_{\mathrm{col}}$, to have its energy sapped by streaming losses, $t_{\text {stream }}$, or to escape from the galaxy via diffusion, $t_{\mathrm{esc}, \mathrm{diff}}$ :

$$
t_{c} \sim\left(t_{\text {col }}^{-1}+t_{\text {stream }}^{-1}+t_{\text {esc, diff }}^{-1}\right)^{-1} .
$$

We can rewrite each of the three ratios appearing inside the parentheses in the above equation in terms of our dimensionless parameters. The collisional loss time is (c.f. equation 11 of Paper I)

$$
t_{\mathrm{col}} \sim \frac{1}{c\left(\rho_{*} / \mu_{p} m_{p}\right) \sigma_{\mathrm{pp}} \eta_{\mathrm{pp}}} \sim \frac{1}{K_{*} \tau_{\mathrm{abs}}}\left(\frac{z_{*}}{\sigma}\right),
$$

where we have dropped factors of order unity, and in the second step we have made use of equation 10 and equation 13 . Similarly, the streaming loss time is (c.f. equation 49 of $\mathrm{Pa}-$ per I)

$$
t_{\text {stream }} \sim \frac{z_{*}}{v_{s}} \sim \frac{1}{K_{*} \tau_{\text {stream }}}\left(\frac{z_{*}}{\sigma}\right),
$$

where in the second step we have used equation 9. Finally, the diffusive escape time is (c.f. equation 47 of Paper I)

$$
t_{\mathrm{esc}, \mathrm{diff}} \sim \frac{z_{*}^{2}}{\kappa_{*}} \sim \frac{1}{K_{*}}\left(\frac{z_{*}}{\sigma}\right),
$$

where we have used equation 11. Inserting these factors into equation 33 for $t_{c}$, and thence into equation 32 , we find

$$
\left(\frac{P_{c}}{P_{*}}\right)_{z=0} \sim f_{\mathrm{Edd}}\left(1+\tau_{\mathrm{abs}}+\tau_{\mathrm{stream}}\right)^{-1} .
$$

Thus if we expect the midplane ratio $P_{C} / P_{*}$ to be of order unity when $f_{\mathrm{Edd}}$ is at the critical value, it follows immediately that

$$
f_{\mathrm{Edd}, \mathrm{c}} \stackrel{\propto}{\sim} \frac{1+\tau_{\mathrm{abs}}+\tau_{\text {stream }}}{\phi_{B}\left(1+f_{\mathrm{gas}}\right)},
$$

where we have now re-inserted the scaling with $\phi_{B}$ and $f_{\text {gas }}$ derived above. This is not, of course, an exact expression, but its scalings are qualitatively correct, as we have seen, and account for the following phenomena: CRs exert a pressure which is i) enhanced by the diffusive nature of their propagation (c.f. Socrates et al. 2008) - this leads to the constant term on the RHS - but attenuated by ii) their collisional and iii) their streaming losses; these lead to the $\propto \tau_{\text {abs }}$ and $\propto \tau_{\text {stream }}$ terms, respectively.

It will be convenient for the remainder of this paper to use our understanding of the scaling behaviour of $f_{\text {Edd,c }}$ to derive an approximate analytic fit that we can use in lieu of the full, numerically-determined solution. We adopt the functional form given by equation 38, and after some numerical experimentation to find coefficients that minimise the error, we arrive at the approximate relationship

$$
f_{\text {Edd, } \mathrm{c}} \approx f_{\text {Edd, } \mathrm{c}, \mathrm{fit}} \equiv \frac{1}{2.4 \phi_{B}\left(1+f_{\mathrm{gas}}\right)}\left(0.26+\frac{\tau_{\mathrm{abs}}}{2.5}+\frac{\tau_{\text {stream }}}{0.31}\right) .
$$

We plot the relative error in this fit, defined as

$$
\text { rel. err. } \equiv \frac{\left|f_{\mathrm{Edd}, \mathrm{c}}-f_{\mathrm{Edd}, \mathrm{c}, \mathrm{fit}}\right|}{f_{\mathrm{Edd}, \mathrm{c}}},
$$

in Figure 3. The figure demonstrates that our approximation is accurate to $\lesssim 20$ percent for $\tau_{\text {abs }}<10$ and $f_{\text {gas }}=0.01-0.99$.
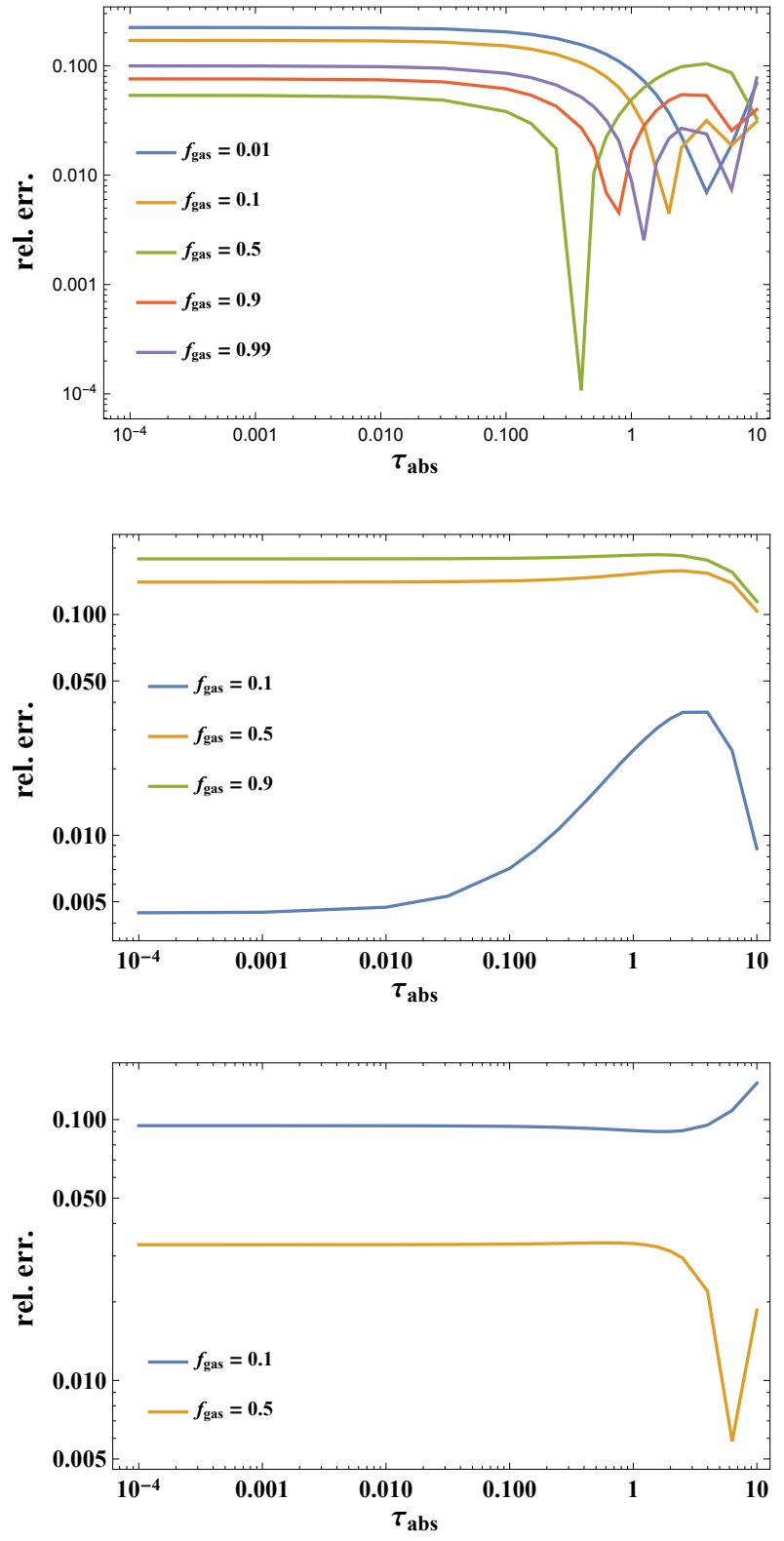

Figure 3. Relative error between the analytic approximation and the full numerically-determined CR critical Eddington ratio. From top to bottom, the panels are for $\tau_{\text {stream }}=\left\{1^{3}, 1.3^{3}, 1.5^{3}\right\}$; gas fractions are as labelled in each panel's legend.

\subsection{The cosmic ray stability limit for star-forming galaxies}

The final step in our calculation is to translate our stability limit from dimensionless $\left(f_{\mathrm{Edd}}, \tau_{\mathrm{abs}}, \tau_{\text {stream }}\right)$ to the physical variables describing a star-forming galactic disc. In particular, we are interested in the highest star formation rate (since star formation produces supernovae that are the primary source of CRs) that a disc can sustain before it becomes unstable to the development of CR-driven outflows.

The conversion from dimensionless to physical variables is straightforward. Given a gas surface density $\Sigma_{\text {gas }}$, velocity dispersion $\sigma$, gas fraction $f_{\text {gas }}$, Alfvén Mach number $M_{A}$, and 
either (depending on our choice of CR transport model) an ionisation fraction $\chi$ or CR energy $E_{\mathrm{CR}}$, we can compute the corresponding $\tau_{\text {stream }}$ and $\tau_{\text {abs }}$ values from equation 22 and equation 23 (for our fiducial streaming model), equation 26 and equation 27 (for the scattering transport model), or equation 30 and equation 31 (for the constant model). From these values plus $f_{\text {gas }}$ we can compute the critical Eddington ratio $f_{\text {Edd,c }}$ using either the numerical procedure outlined in Section 3.1, or, with much less computational expense, our approximate fitting formula (equation 39).

We can obtain a corresponding star formation rate per unit area $\dot{\Sigma}_{\star}$ from this as follows. First, following Paper I, we write the CR flux as

$$
F_{c, 0}=\epsilon_{c, 1 / 2} \dot{\Sigma}_{\star},
$$

where $\epsilon_{c, 1 / 2}$ is the energy injected into CRs in each galactic hemisphere per unit mass of stars formed. We adopt a fiducial value $\epsilon_{c, 1 / 2} \approx 5.6 \times 10^{47} \mathrm{erg} M_{\odot}^{-1}$, which corresponds to assuming Chabrier (2005) initial mass function, that all stars with mass $\geqslant 8 M_{\odot}$ end their lives as supernovae with total energy $10^{51} \mathrm{erg}$, and that $10 \%$ of this SN energy is eventually injected into CRs. Substituting equation 41 into the definition of $f_{\text {Edd }}$ (equation 17 ), we have

$$
\begin{aligned}
\dot{\Sigma}_{\star} & =\frac{F_{*}}{\epsilon_{c, 1 / 2}} K_{*} \beta f_{\text {Edd }}=\frac{\pi G}{\epsilon_{c, 1 / 2}} \frac{\Sigma_{\text {gas }}^{2} \sigma}{f_{\text {gas }}} K_{*} f_{\text {Edd }} \\
& =4.9 \times 10^{-4} \frac{\Sigma_{\text {gas }, 1}^{2} \sigma_{1}}{f_{\text {gas }}} K_{*} f_{\text {Edd }} M_{\odot} \mathrm{pc}^{-2} \mathrm{Myr}^{-1} .
\end{aligned}
$$

By plugging our value of $f_{\text {Edd,c }}$ into equation 42 , together with the appropriate value of $K_{*}$ for our chosen CR transport model (equation 20, equation 24, or equation 28), we obtain the critical star formation rate $\dot{\Sigma}_{\star, c}$ above which galaxies become unstable to CRs.

In order to actually plot $\dot{\Sigma}_{\star, \mathrm{c}}$ versus $\Sigma_{\text {gas }}$, we require values of $\sigma, f_{\text {gas }}$, and $\chi$, which vary systematically with $\Sigma_{\text {gas }}$ on average (e.g., higher surface density galaxies tend to have higher velocity dispersion), but which are not single-valued functions of $\Sigma_{\text {gas }}$ either. To avoid a proliferation of curves, we adopt the same strategy as in Paper I: we interpolate between plausible values of these parameters as a function of $\Sigma_{\text {gas }}$. Specifically, we adopt

$$
\begin{aligned}
f_{\text {gas }}\left(\Sigma_{\text {gas }}\right) & \equiv 0.11 \Sigma_{\text {gas }, 1}^{0.32} \\
\chi\left(\Sigma_{\text {gas }}\right) & \equiv 0.013 \Sigma_{\text {gas }, 1}^{-0.79} \\
\sigma\left(\Sigma_{\text {gas }}\right) & \equiv 8.5 \Sigma_{\text {gas }, 1}^{0.39} \mathrm{~km} / \mathrm{s} .
\end{aligned}
$$

We emphasise that these are not intended to be accurate fits; they are simply intended to provide smooth functions we can use to reduce the multidimensional parameter space of $\Sigma_{\text {gas }}, \sigma, f_{\text {gas }}$, and $\chi$ to a single dimension so that we can represent it on a plot.

With this understood, we plot $\dot{\Sigma}_{\star, \mathrm{c}}$ as a function of $\Sigma_{\text {gas }}$ in Figure 4. We show curves for the cases of i) our fiducial streaming model for CR transport (blue), ii) the alternative scattering (yellow), and iii) the case of constant $\kappa$ (green); for all of these modes we show both results for both $\mathcal{M}_{A}=2$ (solid) and $\mathcal{M}_{A}=1$ (dashed). For any particular curve, the stable region is below and to the right, while the unstable region is above and to the left. Figure 4 also shows a selection of observed galaxies culled from the literature (see Paper I for details of the data compilation), with some particularly significant galaxies shown by the red points: the Milky Way datum ('MW'), its Central Molecular Zone (CMZ), and the nearby starbursts NGC253, M82, and Arp 220, whose $\gamma$-ray emission we modelled in Krumholz et al. (2020).

\section{IMPLICATIONS}

Figure 4 is the central result of this paper. Here we discuss its implications, and explore the physical origin of the result and its sensitivity to a variety of assumptions and parameter choices that we have made.

\subsection{For which galaxies can CRs drive outflows from the star-forming ISM?}

We start by examining our fiducial CR trasnport model, indicated by the blue lines in Figure 4. An immediate conclusion we can draw is that, for physically-plausible scalings of the parameters, the CR stability curve patrols a region very close to the top of the occupied part of the $\left(\Sigma_{\text {gas }}, \dot{\Sigma}_{\star}\right)$ plane for star-forming galaxies with low gas surface densities typical of the Galaxy and local dwarfs. This correspondence strongly suggests that CR feedback on the neutral gas may be an important mechanism in such galaxies: it might limit the ability of galaxies to make excursions above the locus where most of them like to be, or it might be responsible for launching winds and ejecting gas in galaxies that do wander upwards to higher star formation rates.

Conversely, it is evident that, at the higher gas surface densities encountered in local starbursts and high-redshift star-forming galaxies, all the critical curves (not just the one for our fiducial model) diverge away from the observed distribution of galaxies. This implies that CRs cannot drive winds in these systems (cf. Paper I). Mathematically, such a divergence must occur for the following reason: from equation 42 , for the range of $\tau_{\text {abs }}$ for which $f_{\text {Edd,c }} \sim$ const at fixed $\tau_{\text {stream }}$ (cf. Figure 2) we have shown that the critical star formation rate surface density scales approximately as $\dot{\Sigma}_{\star, \mathrm{c}} \underset{\sim}{\sim} \tau_{\text {abs }}^{2} \propto \Sigma_{\text {gas }}^{2}$. On the other hand, the observed surface density of star formation rises with gas surface density with an index $<2$. Physically, the divergence occurs because the high gas number densities in starburst systems kill CRs quickly, meaning that the energy density/pressure they represent cannot build up to be anything comparable to hydrostatic pressures (cf Lacki et al. 2011; Thompson \& Lacki 2013; Crocker et al. 2020a). On the other hand, such a situation constitutes a recipe for CR calorimetry, so these systems are expected - indeed, directly inferred, in a limited number of cases - to be good hadronic $\gamma$-ray sources (cf. Torres et al. 2004; Thompson et al. 2007; Lacki et al. 2010, 2011; Yoast-Hull et al. 2016; Peretti et al. 2019; Krumholz et al. 2020).

\subsection{Instability of galaxies under scattering}

A second significant point that is evident from Figure 4 is that, for the cases of both constant diffusivity and scattering, the critical curves cut well into the occupied region of parameter space for lower surface gas density galaxies; many such galaxies, including the Milky Way, are unstable under 


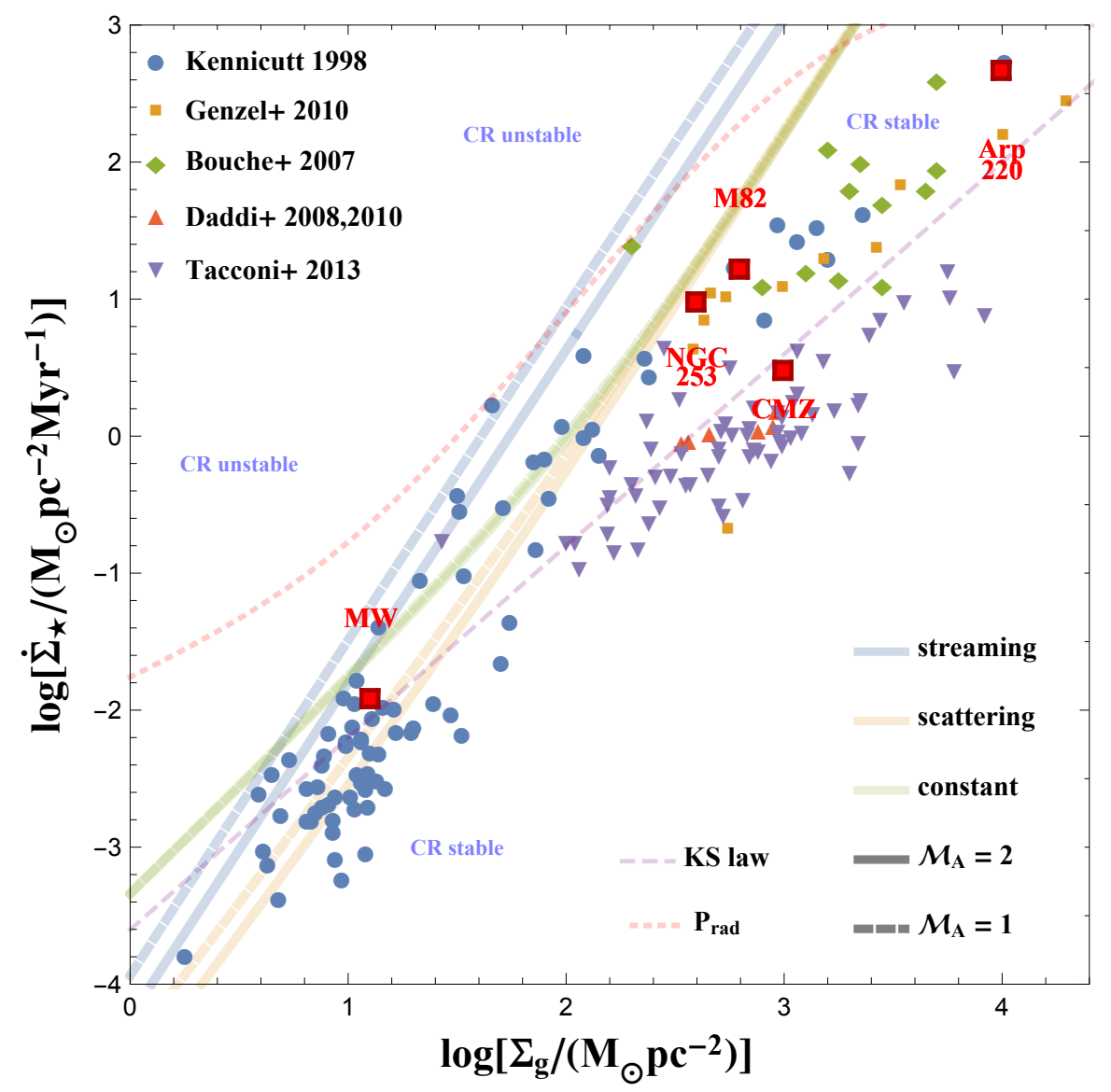

Figure 4. Thick coloured lines show critical stability curves $\dot{\Sigma}_{\star, \mathrm{c}}$, i.e., the star formation rate per unit area at which CR pressure precludes hydrostatic equilibrium, computed assuming one of three different $\mathrm{CR}$ transport modes as indicated (with streaming constituting our fiducial model), and for $\mathcal{M}_{A}=2$ (solid) and $\mathcal{M}_{A}=1$ (dashed) together with fiducial parameter choices for all models, and using the fits for $f_{\mathrm{gas}}, \chi$, and $\sigma$ as a function of $\Sigma_{\text {gas }}$ given by equation 43 - equation 45 . The dashed, diagonal, purple line is the Kennicutt (1998) star formation scaling. The dotted red line is the critical star formation rate surface density obtained for radiation pressure feedback. This curve smoothly interpolates from the single-scattering case (adopting a critical Eddington ratio 0.6 on the basis of the calculation by Wibking, Thompson, \& Krumholz (2018) and assuming a fixed flux-mean dust opacity per mass of dustâĂSgas mixture of $\kappa=1000$ $\mathrm{cm}^{2} / \mathrm{g}$ ) into the regime where the atmosphere is optically thick to FIR radiation due to the dust opacity (Crocker et al. 2018a) with a cross-over at $\Sigma_{\text {gas }} \simeq 10^{3} M_{\odot} / \mathrm{pc}^{2}$ (and we have assumed a young, $<7 \mathrm{Myr}$ old, stellar population for the optically thick part of the curve). Finally, points show observations drawn from the following sources: local galaxies from Kennicutt (1998), z $\sim 2$ sub-mm galaxies from Bouché et al. (2007), and galaxies on and somewhat above the star-forming main sequence at $\mathrm{z} \sim 1-3$ from Daddi et al. (2008, 2010b); Genzel et al. (2010); Tacconi et al. (2013). The red data points show the Solar neighborhood ('MW') and the Central Molecular Zone ('CMZ') of the Milky Way, and three, local starbursts whose $\gamma$-ray emission is modelled in Krumholz et al. (2020). The observations have been homogenised to a Chabrier (2005) IMF and the convention for $\alpha_{\text {CO }}$ suggested by Daddi et al. (2010a); see Krumholz et al. (2012) for details.

this scenario. Since the constant model is not physically wellmotivated, this is not particularly surprising; the more surprising result is for the scattering model. We remind readers that this model for CR transport applies in an environment where there is an extrinsic turbulence cascade that reaches down to the gyroradius scale of the energetically-dominant $\sim \mathrm{GeV}$ CRs. We have shown Krumholz et al. (2020) that this is not the case for the neutral medium that dominates the mass and forms the stars. On the other hand, the longstanding classical interpretation of the totality of the CR and diffuse gamma-ray emission phenomenology is that the spectrum of the Milky Way's steady state, hadronic cosmic ray distribution is informed by exactly this process of scattering on extrinsic turbulence (e.g., Jones et al. 2001).
This is not necessarily a contradiction: unlike the situation for starbursts (Krumholz et al. 2020), the MW midplane ISM is not single phase. Rather, the filling factors of the dense, neutral phase and the more diffuse, ionised phase are similar in the midplane (Kalberla \& Kerp 2009). Moreover, the ionised gas filling factor increases towards unity as we rise away from the midplane and individual SNRs or stellar cluster superbubbles form chimneys into the hot, ionised halo. Thus some - potentially large - fraction of CRs accelerated by SNR shocks in Milky Way-like conditions may never encounter a large grammage of matter in escaping the midplane, i.e., they experience an effective $\tau_{\text {abs }} \ll 1$ and, incidentally, also $\tau_{\text {stream }} \ll 1$ given that streaming losses are generically small, in relative terms, for the scattering mode. 
These CRs - provided that the classical picture of scattering on an extrinsic turbulent cascade is roughly correct will render the ionised gas column hydrostatically unstable. This applies for Milky Way conditions according to Figure 4. Of course, an assumption here is that the classical picture of scattering on extrinsic turbulence is essentially the correct one for the ionised phase, and this may not actually hold (e.g., Zweibel 2017; Blasi 2019). On the other hand, recognising that the ionised gas column for most low surfacedensity galaxies will only constitute some $\lesssim 10 \%$ of the total column, Figure 4 may actually tend to exaggerate stability with respect to cosmic ray feedback in the ionised phase for such galaxies.

In summary, as has long been recognised (Jokipii 1976; Ko et al. 1991; Breitschwerdt et al. 1991; Everett et al. 2008; Socrates et al. 2008), it is hard to escape the conclusions that for local galaxy conditions, CRs will likely drive winds in the ionised gas phase. This will lead to mass loss over cosmological timescales. However, none of these considerations preclude the existence of a hydrostatic equilibrium in the dense, neutral phase that allows it to sustain the star formation process. Overall, the picture we thus arrive at here is that there are effectively two transport regimes operating for CRs in Milky Way-like galaxies (according to the ISM phase within which CRs are propagating). Qualitatively this agrees with the long-standing argument (Ginzburg et al. 1980) that the correct interpretation of local CR phenomenology ${ }^{7}$ is that there are distinct disc and halo CR propagation zones, with the halo diffusion coefficient significantly (i.e., $3-10 \times$ ) larger than the disc one (while the characteristic matter density in the disc is, of course, substantially larger than that in the halo).

\subsection{The role of the Alfvénic Mach number}

One of the important parameters that appears in our models is the Alfvénic Mach number of the turbulence. This parameter controls the streaming speed and thus the strength of streaming losses directly, and also affects the overall diffusion rate - weakly for the scattering or constant models, strongly for the streaming transport model. We have argued based on dynamo theory that $M_{A}$ will always be in the range $\sim 1-2$ in galactic discs (Federrath et al. 2014; Federrath 2016), but it is important to investigate to what extent our conclusions are dependent on this argument.

We first investigate this in the context of the streaming model, where the $M_{A}$-dependence is greatest. In Figure 5 we show critical stability curves for this model computed with various value of $M_{A}$. In the high $M_{A}$ limit the stability curve becomes universal (i.e., independent of the transport mode) because the CR energy density is set purely by hadronic losses right across the range of gas surface density; the critical curve in this limiting case is shown as the dashed red

\footnotetext{
7 Specific aspects of CR phenomenology that support the existence of a CR halo include i) the very low levels of CR anisotropy and ii) the difficulty encountered in otherwise reconciling CR age measurements obtained with unstable "clock" nuclei (like ${ }^{10} \mathrm{Be}$ ) with the grammage encountered by typical $\sim \mathrm{GeV}+\mathrm{CRs}$ as inferred from secondary to primary CR nuclei ratios.
}

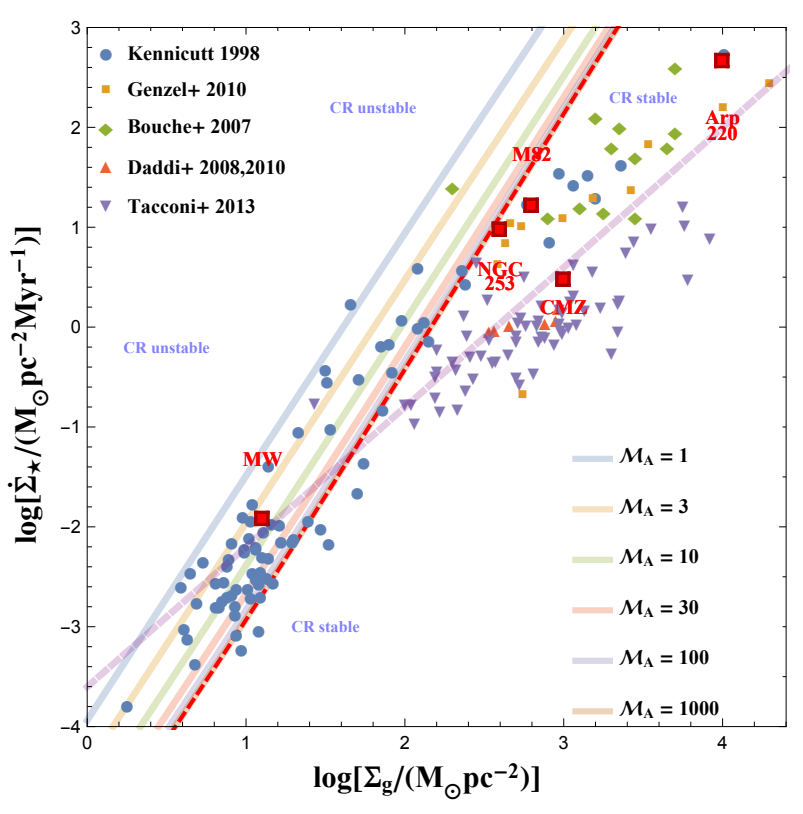

Figure 5. Critical stability curves for the streaming mode of CR transport with $M_{A}$ as given in the legend. Parameters and data points are identical to those used in Figure 4, except for $\boldsymbol{M}_{A}$. The red, dashed curve shows the stability curve in the high $M_{A}$ limit, where it is completely determined by hadronic losses.

line in the figure. Note that, were ordinary, local disc galaxies operating in this limit, their neutral ISM phase would be unstable and driving a strong outflow, something that we do not observe. This implies, minimally, that magnetic fields in such galaxies are not too far below equipartition with respect to turbulent energy density (as expected in the case that a local turbulent dynamo is operating, and as we see directly in the Milky Way). More speculatively, it may be that gas motions induced by CRs in the case that the atmosphere is CR unstable help to drive magnetic fields towards equipartition at the lower surface gas density end of the distribution.

The difference between $M_{A}=1$ and 2 is far smaller for the scattering and constant models. This occurs because, for these modes, the streaming loss timescales are relatively long while the diffusive escape or collisional timescales are either mildly dependent or completely independent of $M_{A}$. The critical curves for the scattering and constant $\kappa$ cases also become identical at high $\Sigma_{\text {gas }}$. This occurs because the collisional timescale formula is universal and collisional losses are solely responsible for setting the CR energy density in this regime. The streaming curve does not exhibit this behaviour because here streaming losses remain comparable to collisional losses even up to very high $\Sigma_{\text {gas }}$. The streaming case approaches the streaming and constant curves at high $\Sigma_{\text {gas }}$ only in the limit $M_{A} \gg 1$.

\subsection{Unimportance of convective transport}

On the basis of equation $42, \dot{\Sigma}_{\star, \mathrm{c}} \propto K_{*} f_{\text {Edd,c }}$, so it might seem that we should expect that there should be clear structures in the critical curves where $K_{*}$ becomes equal to unity 
and the CR transport mechanism changes between convection and some other process. The $K_{*}=1$ regime occurs for $\Sigma_{\text {gas }} \lesssim 10 M_{\odot} \mathrm{pc}^{-2}$ for the streaming transport model, and for $\Sigma_{\text {gas }} \gtrsim 10^{3} M_{\odot} \mathrm{pc}^{-2}$ for the starburst and scattering transport models, yet there are clearly no sharp features visible in the critical curves. Indeed, even if we explicitly ignore convection (i.e., we allow $K_{*}<1$ when computing $\dot{\Sigma}_{\star, \mathrm{c}}$ ), we find critical curves that are essentially identical.

We can understand the reason for this both mathematically and physically. Mathematically, for a scattering or constant $\kappa$ model of CR transport, the diffusive transport rate only becomes smaller than the convective rate in galaxies with very high surface densities. However, these galaxies also have $\tau_{\mathrm{abs}} \gg 1$, and in this limit we have $f_{\mathrm{Edd}, \mathrm{c}} \propto \tau_{\mathrm{abs}}$. Since $\tau_{\mathrm{abs}}=\tau_{\mathrm{pp}} / K_{*} \beta$, we arrive at $\dot{\Sigma}_{\star} \propto \tau_{\mathrm{pp}} / \beta$, with no dependence on $K_{*}$; this is why a sharp change in the value of $K_{*}$ does not generate a corresponding sharp change in $\dot{\Sigma}_{\star}$. Physically, the origin of this behaviour is that the only galaxies in which the rate of diffusive CR transport becomes smaller than the rate of convective transport are those with very high gas densities, and thus high $\tau_{\text {abs }}$. In these galaxies, the dominant $\mathrm{CR}$ loss process is no longer diffusive escape, it is pion production. In this regime, the rate of transport diffusive or convective - is irrelevant to setting the CR energy density. Instead, the CR energy density is simply set by the competition between injection and pion loss, and it is the balance between these two processes that determines the location of the critical curve, the point at which the CR pressure becomes too high to permit hydrostatic balance.

For the streaming model, the transition to convective transport happens at low gas surface density. However, in these galaxies we also have $\tau_{\text {stream }} \gtrsim 1$, and thus $f_{\text {Edd,c }} \propto$ $\tau_{\text {stream }}$. Since $\tau_{\text {stream }} \propto 1 / K_{*}$, the dependence of $\dot{\Sigma}_{\star}$ on $K_{*}$ again disappears, and thus there is again no sudden change in the critical curve when we reach the convective limit. The physical origin of the behaviour in this place is that, for this transport model, CRs are lost primarily to streaming rather than to escape. We again therefore have a situation where the CR energy density is set by the balance between streaming and injection, a balance that does not depend on the effective diffusion coefficient.

\subsection{Cosmic rays versus radiation pressure as wind launching mechanisms}

CRs are of interest as a feedback mechanism partly because they are much less efficiently lost to cooling than hot gas produced by SN explosions. However, a second appealing aspect of CR feedback is that CRs are "cool", in that they can accelerate gas without the need for a shock, and thus naturally explain the presence of low-temperature species in galactic winds. It is therefore interesting to compare CRs to radiation pressure, which is another cool feedback mechanism. Radiation pressure can be delivered either by the direct stellar radiation field or by radiation that has been absorbed by dust and reprocessed into the infrared (cf. red dotted line in Figure 4 which interpolates between these limits). At galactic scales, the latter mechanism is only important in the densest starbursts (Thompson et al. 2005; Crocker et al. 2018a,b), precisely where we have shown that CR feedback is ineffective. The more interesting comparison is therefore in the regime of low surface density galaxies, where direct, or "single scattering", radiation pressure dominates (e.g., Scoville et al. 2001; Murray et al. 2005; Fall et al. 2010; Andrews \& Thompson 2011; Thompson et al. 2015; Skinner \& Ostriker 2015; Thompson \& Krumholz 2016; Wibking, Thompson, \& Krumholz 2018).

For a region of a galactic disc with areal star formation rate $\dot{\Sigma}_{\star}$, the momentum per unit area per unit time delivered by the radiation field per hemisphere in the single scattering limit is

$$
\dot{\Pi}_{\mathrm{Ss}}=\frac{1}{c} \dot{\Sigma}_{\star} \Phi_{1 / 2}
$$

where

$$
\Phi_{1 / 2} \simeq 6.0 \times 10^{50} \mathrm{erg} / M_{\odot}
$$

is the efficiency for conversion of gas mass into radiation (into one galactic hemisphere) via the star formation process (Kennicutt \& Evans 2012). Note that we are assuming that all of the direct stellar radiation is absorbed. This is an upper limit, but cannot be wrong by a large factor, since, as pointed out by Andrews \& Thompson (2011), 1/3 of the radiation momentum budget is in ionising photons, which will be absorbed even by a tiny column of neutral gas. By comparison, the momentum per unit area per unit time in the upward direction delivered by CRs into each hemisphere, integrating over the gas column, is

$$
\dot{\Pi}_{c}=-P_{*} \int_{0}^{\infty} \frac{d p_{c}}{d \xi} d \xi .
$$

To evaluate the integral, we can make use of equation 1 :

$$
\begin{aligned}
\int_{0}^{\infty} \frac{d p_{c}}{d \xi} d \xi & =\frac{1}{\tau_{\text {stream }}}\left[\int_{0}^{\infty}\left(\tau_{\text {abs }} r p_{c}+\frac{\tau_{\text {stream }}}{\beta_{S}} \frac{d \mathcal{F}_{c}}{d \xi}\right) d \xi\right] \\
& =\frac{1}{\beta_{s}}\left[\mathcal{F}_{c}(\infty)-\mathcal{F}_{c}(0)\right]+\frac{f_{\text {Edd }} f_{\text {cal }}}{\tau_{\text {stream }}}
\end{aligned}
$$

where

$$
f_{\text {cal }}=\frac{\tau_{\mathrm{abs}}}{f_{\mathrm{Edd}}} \int_{0}^{\infty} r p_{c} d \xi
$$

is the "calorimetric fraction", i.e., the fraction of all CRs that are lost to pion production (c.f. equation 64 of Paper I), and $\mathcal{F}_{c}(\infty)$ is the flux at $\xi=\infty$.

Therefore we find that the ratio of $\mathrm{CR}$ to singlescattering radiation momentum is

$$
\begin{aligned}
\frac{\dot{\Pi}_{c}}{\dot{\Pi}_{\mathrm{ss}}} & =\left(\frac{\epsilon_{c, 1 / 2}}{\Phi_{1 / 2}}\right) \frac{1}{\beta_{s}}\left[1-f_{\text {cal }}-\frac{\mathcal{F}_{c}(\infty)}{\mathcal{F}_{c}(0)}\right] \\
& \simeq 9.3 \times 10^{-4} \frac{1}{\beta_{s}}\left[1-f_{\text {cal }}-\frac{\mathcal{F}_{c}(\infty)}{\mathcal{F}_{c}(0)}\right] \\
& \simeq \frac{2.8}{v_{s, 2}}\left[1-f_{\text {cal }}-\frac{\mathcal{F}_{c}(\infty)}{\mathcal{F}_{c}(0)}\right]
\end{aligned}
$$

where $v_{s, 2} \equiv v_{s} /(100 \mathrm{~km} / \mathrm{s})$ and we have made use of equation 9 , equation 17 , equation 41 to simplify. This expression has a straightforward physical interpretation. The leading numerical factor of $\approx 10^{-3}$ in the second line represents the ratio of energy injected into photons versus energy injected into CRs. The second term, $1 / \beta_{s}$, which is always $\gg 1$, accounts for the fact that CRs transfer momentum to the gas much more efficiently than photons, due to the fact that 
their propagation speed is limited to a value $\ll c$ by scattering off Alfvén waves. Finally, the factor in square brackets just represents the reduction in $\mathrm{CR}$ momentum transfer due to loss of CRs by pion production (the $f_{\text {cal }}$ term) and due to the escape of some fraction of the injected CRs from the disc without interaction (the $\mathcal{F}_{c}(\infty) / \mathcal{F}_{c}(0)$ term). It approaches unity if all of the CR energy is lost to streaming, and becomes smaller if there is significant CR energy loss into other channels. This term can be evaluated numerically from our solutions, and, for the low surface density galaxies with which we are concerned here, is generally in the range $\sim 0.1-1-$ see Section 4.3 of Paper I.

The implication of equation 51 is that CRs deliver more momentum to the gas than single-scattering radiation pressure if the CR streaming speed satisfies $v_{S} \lesssim 100 \mathrm{~km} \mathrm{~s}^{-1}$; the exact condition will depend on details such as the fraction of photon momentum that is actually absorbed, which is likely close to unity in spiral galaxies, but below unity in dust-poor dwarfs. Regardless of the exact numerical limit on $v_{s}$, the condition is certainly met if the CRs propagate through ionised gas, for which the streaming speed is nearly equal to the total gas Alfvén speed, which, for $M_{A} \sim 1$, is comparable to the $\sim 10 \mathrm{~km} \mathrm{~s}^{-1}$ velocity dispersion in the ISM. Thus in the scattering or constant $\kappa_{*}$ CR propagation scenarios, CRs are more important than photons.

For our favoured streaming scenario, the question of whether CRs or photons are more important is more subtle because the streaming speed in this case is close to the ion Alfvén velocity, which, in a weakly-ionised medium, is much larger than the total Alfvén velocity or the velocity dispersion. Since we are concerned here with low surface density galaxies whose interstellar media are predominantly atomic, we expect the ionisation fraction $\chi \sim 10^{-2}$ (Wolfire et al. 2003), and thus the ion Alfvén speed to be $\approx 10$ times the bulk gas velocity dispersion. This suggests that CRs and single scattering radiation are of roughly comparable importance, and both may contribute to the launching of galactic winds in such galaxies (cf. Figure 4). CRs are probably somewhat more important than photons in low-metallicity dwarfs, where the absence of dust will render galaxies more transparent and thus reduce $\dot{\Pi}_{\mathrm{ss}}$, though only by a factor of $\sim 3$ as noted above; on the other hand, it is possible that the equilibrium ionisation fraction is also slightly higher in lowmetallicity dwarfs. ${ }^{8}$ For denser galaxies whose interstellar media are largely molecular, $\chi$ is smaller, and the ion Alfvén speed correspondingly larger. In these galaxies photons deliver more momentum than CRs; however, this changeover likely has little practical importance, since both direct photons and CRs are generally unimportant in these galaxies.

Finally, we note that our equation 51 is somewhat different from the analogous expression (their equation 21) of Socrates et al. (2008). We discuss the reasons for this difference in Appendix A.

8 Also note that, though the (red, dashed) $P_{\text {rad,ss }}$ line in Figure 4 falls above the locus of points at low surface densities, Thompson \& Krumholz (2016) argue that radiation pressure in a turbulent medium will be most important along low-column density sightlines not representative of the mean gas surface density.

\section{CONCLUSIONS}

In this paper we analyse the stability of the neutral, starforming phase of galactic discs against cosmic ray (CR) pressure. We use an idealised model where such discs are taken to be plane-parallel slabs of gas confined by stellar and gas self-gravity, and supported by a combination of turbulent and CR pressure. Such a system is characterised primarily by three dimensionless numbers: the effective optical depths of the disc to $\mathrm{CR}$ absorption (via $\pi$ production) and to $\mathrm{CR}$ streaming, and the CR Eddington ratio (defined by the ratio of the CR momentum flux to the gravitational momentum flux). The primary result of our analysis is that such a system possesses a stability limit: for a given effective optical depth, there exists a maximum CR Eddington ratio above which the system cannot remain hydrostatic. While the nature of the non-linear development of the resulting instability is uncertain, studies of the analogous instability driven by radiation suggests that the result is likely to be a outflow that removes mass until the system is driven back below the stability limit.

Given standard estimates for the efficiency with which $\mathrm{SNe}$ inject CRs into galaxies, together with characteristic numbers describing the magnetohydrodynamic turbulence in the ISM and a model for CR transport in a turbulent medium, we can translate our stability limit directly into a line in the space of gas surface density and star formation rate, the so-called Kennicutt-Schmidt (KS) plane. We find that the stability limit projected on to the KS plane is close to a line of slope 2, which, for our favoured model of CR transport closely matches the upper envelope of observed systems with the surface densities characteristic of modern spiral and dwarf galaxies, $\Sigma_{\text {gas }} \lesssim 300 M_{\odot} \mathrm{pc}^{-2}$. While a scaling $\propto \Sigma_{\text {gas }}^{2}$ for the critical star formation rate density with gas surface density is generic to feedback mechanisms (e.g. Andrews \& Thompson 2011), the fact that our calculation should have produced such a coincidence between the normalization of the critical curve and the upper range of the occupied KS parameter space is surprising: In the dimensionless parameter space of $\tau_{\text {stream }}, \tau_{\text {abs }}$, and $f_{\text {Edd }}$ that defines our system, the critical value of $f_{\text {Edd }}$ above which the gas column is rendered hydrostatically unstable follows purely from the mathematical form of our ODEs. The only astrophysical inputs required to map this to the KS plane are then fundamental constants (e.g., the $p p$ cross-section), quantities describing general physical processes that are unrelated to galaxies (e.g., the saturation field strength of turbulent dynamos), and quantities describing microphysical processes such as the conversion efficiency from supernova kinetic energy to CR energy. The only complex modeling needed is that required to estimate the ionization fraction, which is determined at least partly by the CRs themselves. Given these inputs, the overall similarity of the CR stability limit to the observed galaxy distribution seems unlikely to be a coincidence. We suggest that the star-forming gas in modern and/or low surface gas density galaxies is poised close to instability such that rather small changes in ISM parameters imply the launching of CR-driven outflows; CRs - possibly in concert wtih direct radiation pressure - thus define the upper limit to the star-formation efficiency of ordinary, starforming disc galaxies.

In contrast, we find that galaxies with higher gas and 
star formation surface densities lie well below the CR stability limit. This divergence between the CR stability line and the sequence occupied by observed galaxies has two related but distinct causes. The first is simply that the fundamental scaling that governs all considerations of feedback: the selfgravitational pressure of a galactic disc rises as the square of the gas surface density, whereas the available energy input from star formation, given that the observed index of the Kennicutt-Schmidt relation is $<2$, rises more slowly. However, this alone would not be enough to prevent CRs from becoming significant at high surface densities, since, in the absence of loss mechanisms, CRs would also become increasingly well-confined in high surface density galaxies, and this would cause a superlinear rise in the CR pressure. Indeed, it was precisely this consideration that led Socrates et al. (2008) to conclude that CR feedback dominates in high surface-density galaxies. That it does not do so is due to the second factor that suppresses CR feedback in gas-rich galaxies: the increasing importance of hadronic losses. We show that the critical Eddington ratio above which CRs destabilise a galactic disc scales as the sum of the optical depths of a galactic disc to streaming and hadronic losses. While the former varies only weakly across the star-forming sequence, the latter becomes very large in high surface-density galaxies. Consequently, despite the fact that the discs are starburst galaxies that confine CRs quite well, hadronic losses prevent the CR energy density from building up to the point where CRs are able to launch outflows. Thus we conclude that CRs cannot be dynamically important in the star-forming ISM phase of these galaxies. Conversely, however, due to the importance of pion losses, these galaxies are good CR calorimeters and, therefore, $\gamma$-ray sources (cf. Torres et al. 2004; Thompson et al. 2007; Lacki et al. 2010, 2011; Yoast-Hull et al. 2016).

In future work we intend to explore the consequences of the picture set out here and in Krumholz et al. (2020) and Paper I for understanding the far infrared-radio continuum correlation and the emerging far infrared- $-\gamma$-ray correlation, and to delimit the possible contribution of hadronic $\gamma$-ray emission from star-forming galaxies to the isotropic $\gamma$-ray flux as predicted by our model.

\section{DATA AVAILABILITY STATEMENT}

No new data were generated or analysed in support of this research.

\section{ACKNOWLEDGEMENTS}

This research was funded by the Australian Government through the Australian Research Council, awards FT180100375 (MRK) and DP190101258 (RMC and MRK). RMC gratefully acknowledges conversations with Felix Aharonian, Geoff Bicknell, Yuval Birnboim, Luke Drury, Alex Lazarian, Chris McKee, Christoph Pfrommer, Heinz Völk, and Siyao Xu. MRK and TAT acknowledge support from the Simons Foundation through the Simons Symposium Series "Galactic Superwinds: Beyond Phenomenology", during which some aspects of this work were planned. TAT thanks
Brian Lacki and Eliot Quataert for discussions and collaboration. TAT is supported in part by National Science Foundation Grant \#1516967 and NASA ATP 80NSSC18K0526.

\section{REFERENCES}

Abdo, A. A., Ackermann, M., Ajello, M., et al. 2010, ApJ, 709, L152

Acero, F., Aharonian, F., Akhperjanian, A. G., et al. 2009, Science, 326, 1080

Andrews, B. H., \& Thompson, T. A. 2011, ApJ, 727, 97

Beck, R. 2015, A\&ARv, 24, 4

Behroozi, P. S., Wechsler, R. H., \& Conroy, C. 2013, ApJ, 762, L31

Bell, A. R. 1978, MNRAS, 182, 147

Bell, A. R. 2004, MNRAS, 353, 550

Blasi, P. 2019, Galaxies, 7, 64

Boettcher, E., Zweibel, E. G., Gallagher, J. S., III, \& Benjamin, R. A. 2016, ApJ, 832, 118

Booth, C. M., Agertz, O., Kravtsov, A. V., \& Gnedin, N. Y. 2013, ApJ, 777, L16

Bouché, N., Cresci, G., Davies, R., et al. 2007, ApJ, 671, 303

Boulares, A., \& Cox, D. P. 1990, ApJ, 365, 544

Breitschwerdt, D., McKenzie, J. F., \& Voelk, H. J. 1991, A\&A , 245, 79

Breitschwerdt, D., McKenzie, J. F., \& Voelk, H. J. 1993, A\&A , 269, 54

Buck, T., Pfrommer, C., Pakmor, R., et al. 2019, arXiv e-prints, arXiv:1911.00019

Chabrier, G. 2005, The Initial Mass Function 50 Years Later, 327, 41

Chan, T. K., Kereš, D., Hopkins, P. F., et al. 2019, MNRAS, 488, 3716

Cho, J., \& Lazarian, A. 2002, Phys. Rev. Lett., 88, 245001

Cho, J., \& Lazarian, A. 2003, MNRAS, 345, 325

Ceverino, D., Klypin, A., Klimek, E. S., et al. 2014, MNRAS, 442, 1545

Condon, J. J. 1992, ARA\&A, 30, 575

Cox, D. P., \& Smith, B. W. 1974, ApJ, 189, L105

Crocker, R. M., Jones, D. I., Aharonian, F., et al. 2011, MNRAS, 411, L11

Crocker, R. M. 2012, MNRAS, 423, 3512

Crocker, R. M., Krumholz, M. R., Thompson, T. A., \& Clutterbuck, J. 2018 (CKTC18), MNRAS, 478, 81

Crocker, R. M., Krumholz, M. R., Thompson, T. A., Baumgardt, H., \& Mackey, D. 2018, MNRAS, 481, 4895

Crocker, R. M., Krumholz, M. R., \& Thompson, T. A. [Paper I]

Crutcher, R. M. 1999, ApJ, 520, 706

Daddi, E., Dannerbauer, H., Elbaz, D., et al. 2008, ApJ, 673, L21

Daddi, E., Bournaud, F., Walter, F., et al. 2010, ApJ, 713, 686

Daddi, E., Elbaz, D., Walter, F., et al. 2010, ApJ, 714, L118

Davis, S. W., Jiang, Y. F., Stone, J. M., \& Murray, N. 2014, ApJ, 796,107

Dekel, A., Sarkar, K. C., Jiang, F., Bournaud, F., Krumholz, M. R., Ceverino, D., \& Primack, J. 2019, MNRAS, 4, 4753

Domingo-Santamaría, E., \& Torres, D. F. 2005, A\&A , 444, 403

Dorfi, E. A., \& Breitschwerdt, D. 2012, A\&A , 540, A77

Drury, L. O., Markiewicz, W. J., \& Voelk, H. J. 1989, A\&A , 225, 179

Drury, L. O. '., \& Strong, A. W. 2017, A\&A , 597, A117

Everett, J. E., Zweibel, E. G., Benjamin, R. A., et al. 2008, ApJ, 674,258

Faerman, Y., Sternberg, A., \& McKee, C. F. 2017, ApJ, 835, 52

Fall, S. M., Krumholz, M. R., \& Matzner, C. D. 2010, ApJ, 710, L142

Federrath, C. 2016, J. Plasm. Phys., 82, 535820601 
Federrath, C., Schober, J., Bovino, S., \& Schleicher, D. R. G. 2014, ApJ, 797, L19

Fermi-LAT collaboration, et al. 2012, ApJ, 755, 164

Fermi-LAT collaboration, et al. 2019, submitted to ApJS, arXiv: 1902.10045

Funk, S. 2015, Annual Review of Nuclear and Particle Science, 65,245

Gentry, E. S., Krumholz, M. R., Dekel, A., \& Madau, P. 2017, MNRAS, 465, 2471

Genzel, R., Tacconi, L. J., Gracia-Carpio, J., et al. 2010, MNRAS, 407, 2091

Ghosh, A., \& Ptuskin, V. S. 1983, Ap\&SS, 92, 37

Ginzburg, V. L., \& Syrovatskii, S. I. 1964, The Origin of Cosmic Rays

Ginzburg, V. L., Khazan, I. M., \& Ptuskin, V. S. 1980, Ap\&SS, 68,295

Hanasz, M., Lesch, H., Naab, T., et al. 2013, ApJ, 777, L38

Heckman, T. M., Armus, L., \& Miley, G. K. 1990, ApJS, 74, 833

Heckman, T. M. 2002, Extragalactic Gas at Low Redshift, 254, 292

Heintz, E., \& Zweibel, E. G. 2018, ApJ, 860, 97

Hill, A. S., Joung, M. R., Mac Low, M.-M., et al. 2012, ApJ, 750, 104

Hillas, A. M. 2005, JPGNP, 31, 95

Hopkins, P. F., Quataert, E., \& Murray, N. 2011, MNRAS, 417, 950

Ipavich, F. M. 1975, ApJ, 196, 107

Jiang, Y.-F., \& Oh, S. P. 2018, ApJ, 854, 5

Jokipii, J. R. 1976, ApJ, 208, 900

Jones, F. C., Lukasiak, A., Ptuskin, V., et al. 2001, ApJ, 547, 264

Jubelgas, M., Springel, V., Enßlin, T., \& Pfrommer, C. 2008, A\&A , 481, 33

Kafexhiu, E., Aharonian, F., Taylor, A. M., \& Vila, G. S. 2014, Phys. Rev. D, 90, 123014

Kalberla, P. M. W., \& Kerp, J. 2009, ARA\&A, 47, 27

Kennicutt, R. C., Jr. 1998, ApJ, 498, 541

Kennicutt, R. C., Jr., \& Evans, N. J. E., II. 2012, ARA\&A, 50, 531

Kim, C.-G., \& Ostriker, E. C. 2015, ApJ, 802, 99

Ko, C. M., Dougherty, M. K., \& McKenzie, J. F. 1991, A\&A , 241,62

Kroupa, P. 2001, MNRAS, 322, 231

Krumholz, M. R., \& Dekel, A. 2010, MNRAS, 406, 112

Krumholz, M. R., \& Thompson, T. A. 2012, ApJ, 760, 155

Krumholz, M. R., Dekel, A., \& McKee, C. F. 2012, ApJ, 745, 69

Krumholz, M. R., \& Thompson, T. A. 2013, MNRAS, 434, 2329

Krumholz, M. R., Kruijssen, J. M. D., \& Crocker, R. M. 2017, MNRAS, 466, 1213

Krumholz, M. R., Burkhart, B., Forbes, J. C., \& Crocker, R. M. 2018, MNRAS, 477, 2716

Krumholz, M. R., Crocker, R. M., Xu, S., Lazarian, A., Rosevear, M. T., \& Bedwell-Wilson, J. 2020, MNRAS, in press, (arXiv:1911.09774)

Kulsrud, R. M. 2005, Plasma physics for astrophysics / Russell M. Kulsrud. Princeton, N.J. : Princeton University Press, c2005. (Princeton series in astrophysics),

Lacki, B. C., Thompson, T. A., \& Quataert, E. 2010, ApJ, 717, 1

Lacki, B. C., Thompson, T. A., Quataert, E., Loeb, A., \& Waxman, E. 2011, ApJ, 734, 107

Lacki, B. C. 2013, arXiv:1308.5232

Levermore, C. D., \& Pomraning, G. C. 1981, ApJ, 248, 321

Levermore, C. D. 1984, J. Quant. Spectrosc. Radiat. Transfer, 31, 149

Martin, P. 2014, A\&A , 564, A61

Martizzi, D., Faucher-Giguère, C.-A., \& Quataert, E. 2015, MNRAS, 450, 504

McKee, C. F., \& Ostriker, J. P. 1977, ApJ, 218, 148
McKee, C. F., Parravano, A., \& Hollenbach, D. J. 2015, ApJ, 814, 13

Muratov, A. L., Kereš, D., Faucher-Giguère, C.-A., et al. 2015, MNRAS, 454, 2691

Murray, N., Quataert, E., \& Thompson, T. A. 2005, ApJ, 618, 569

Nomoto, K., Tominaga, N., Umeda, H., et al. 2006, Nuclear Phys. A, 777, 424

Paglione, T. A. D., \& Abrahams, R. D. 2012, ApJ, 755, 106

Pakmor, R., Pfrommer, C., Simpson, C. M., \& Springel, V. 2016, ApJ, 824, L30

Parker, E. N. 1966, ApJ, 145, 811

Peng, F.-K., Wang, X.-Y., Liu, R.-Y., Tang, Q.-W., \& Wang, J.-F. 2016, ApJ, 821, L20

Peretti, E., Blasi, P., Aharonian, F., et al. 2019, MNRAS, 487, 168

Persic, M., Rephaeli, Y., \& Arieli, Y. 2008, A\&A , 486, 143

Pfrommer, C., Pakmor, R., Simpson, C. M., \& Springel, V. 2017, ApJ, 847, L13

Protheroe, R. J., Ott, J., Ekers, R. D., et al. 2008, MNRAS, 390, 683

Ptuskin, V. S., Voelk, H. J., Zirakashvili, V. N., et al. 1997, A\&A , 321, 434

Ptuskin, V. S., Moskalenko, I. V., Jones, F. C., et al. 2006, ApJ, 642, 902

Recchia, S., Blasi, P., \& Morlino, G. 2016, MNRAS, 462, 4227

Recchia, S., Blasi, P., \& Morlino, G. 2017, MNRAS, 470, 865

Rosdahl, J., Schaye, J., Teyssier, R., \& Agertz, O. 2015, MNRAS, 451,34

Ruszkowski, M., Yang, H.-Y. K., \& Zweibel, E. 2017, ApJ, 834, 208

Rybicki, G. B., \& Lightman, A. P. 1979, A Wiley-Interscience Publication

Salem, M., \& Bryan, G. L. 2014, MNRAS, 437, 3312

Salem, M., Bryan, G. L., \& Corlies, L. 2016, MNRAS, 456, 582

Samui, S., Subramanian, K., \& Srianand, R. 2010, MNRAS, 402, 2778

Schlickeiser, R. 2002, Cosmic ray astrophysics / Reinhard Schlickeiser, Astronomy and Astrophysics Library; Physics and Astronomy Online Library. Berlin: Springer. ISBN 3-540-664653, 2002, XV + 519 pp.,

Scoville, N. Z., Polletta, M., Ewald, S., et al. 2001, Astronom. J., 122,3017

Skilling J., 1971, ApJ, 170, 265

Skinner, M. A., \& Ostriker, E. C. 2015, ApJ, 809, 187

Simpson, C. M., Pakmor, R., Marinacci, F., et al. 2016, ApJ, 827, L29

Socrates, A., Davis, S. W., \& Ramirez-Ruiz, E. 2008, ApJ, 687, $202-215$

Strong, A. W., Porter, T. A., Digel, S. W., et al. 2010, ApJ, 722, L58

Tacconi, L. J., Neri, R., Genzel, R., et al. 2013, ApJ, 768, 74

Tennekes, H., \& Lumley, J. L. 1972, First Course in Turbulence, Cambridge: MIT Press, 1972

Thomas, T., \& Pfrommer, C. 2019, MNRAS,

Thompson, T. A., \& Lacki, B. C. 2013, Cosmic Rays in Starforming Environments, 283

Thompson, T. A., Quataert, E., \& Murray, N. 2005, ApJ, 630, 167

Thompson, T. A., Quataert, E., Waxman, E., et al. 2006, ApJ, 645,186

Thompson, T. A., Quataert, E., \& Waxman, E. 2007, ApJ, 654, 219

Thompson, T. A., Fabian, A. C., Quataert, E., \& Murray, N. 2015, MNRAS, 449, 147

Thompson, T. A., \& Krumholz, M. R. 2016, MNRAS, 455, 334

Thornton, K., Gaudlitz, M., Janka, H.-T., \& Steinmetz, M. 1998, ApJ, 500, 95 
Torres, D. F., Reimer, O., Domingo-Santamaría, E., et al. 2004, ApJ, 607, L99

Tsang, B. T. H., \& Milosavljević, M. 2015, MNRAS, 453, 11081120

Uhlig, M., Pfrommer, C., Sharma, M., et al. 2012, MNRAS, 423, 2374

VERITAS Collaboration, Acciari, V. A., Aliu, E., et al. 2009, Nature , 462, 770

Vogelsberger, M., Genel, S., Springel, V., et al. 2014, MNRAS, 444, 1518

Völk, H. J., Aharonian, F. A., \& Breitschwerdt, D. 1996, Space Sci. Rev., 75, 279

Wadepuhl, M., \& Springel, V. 2011, MNRAS, 410, 1975

Wentzel, D. G. 1974, ARA\&A, 12, 71

Wibking, B. D., Thompson, T. A., \& Krumholz, M. R. 2018, MNRAS, 477, 4665

Wiener, J., Pfrommer, C., \& Oh, S. P. 2017, MNRAS, 467, 906

Wilson, C. D., Elmegreen, B. G., Bemis, A., et al. 2019, ApJ, 882, 5

Wolfire, M. G., McKee, C. F., Hollenbach, D., et al. 2003, ApJ, 587,278

Xu S., \& Lazarian A., 2017, New Journal of Physics, 19, 065005

Yan, H., \& Lazarian, A. 2008, ApJ, 673, 942

Yoast-Hull, T. M., Gallagher, J. S., \& Zweibel, E. G. 2016, MNRAS, 457, L29

Zirakashvili, V. N., Breitschwerdt, D., Ptuskin, V. S., et al. 1996, A\&A , 311, 113

Zirakashvili, V. N., \& Völk, H. J. 2006, ApJ, 636, 140

Zweibel, E. G. 2013, Physics of Plasmas, 20, 055501

Zweibel, E. G. 2017, Physics of Plasmas, 24, 055402

\section{APPENDIX A: COSMIC RAYS VERSUS RADIATION: COMPARISON TO THE RESULTS OF SOCRATES ET AL. (2008)}

Our result for the ratio of CR to single-scattering radiation momentum imparted to the gas, equation 51, is substantially different at first glance from that derived by Socrates et al. (2008). In this appendix we explain the reasons for this difference. Using our notation, the basic result from Socrates et al., their equation 21 is

$$
\dot{\Pi}_{c} \sim \tau_{\mathrm{CR}} \epsilon_{c, 1 / 2} \dot{\Sigma}_{\star}
$$

where $\tau_{\mathrm{CR}}$ is the effective optical depth of the galactic disc to CR scattering, which Socrates et al. argue is $\sim 10^{3}$. This expression differs from our equation 51 in that dimensionless factor on the right hand side is $\tau_{\mathrm{CR}}$, rather than $\left[1-f_{\text {cal }}-\right.$ $\left.\mathcal{F}_{c}(\infty) / f_{\text {Edd }}\right] / \beta_{s}$.

The difference in the two expressions can be explained by noting that the expression of Socrates et al. does not incorporate any loss mechanisms for CRs, either streaming or hadronic ${ }^{9}$. Thus they are here implicitly taking the limits $\tau_{\text {abs }} \rightarrow 0$ and $\tau_{\text {stream }} \rightarrow 0$. We can first verify that, if we adopt the same limit, our results reduce to theirs. In this case we cannot use equation 51 directly, because in this limit $f_{\text {cal }} \rightarrow$ $0, \mathcal{F}_{c}(\infty) \rightarrow f_{\text {Edd }}$, and $\beta_{s} \rightarrow 0$, and thus the numerator and denominator of the equation both approach zero. However, for the case of zero losses, equation 1 immediately implies $d \mathcal{F}_{c} / d \xi=0$, so $\mathcal{F}_{c}=\mathcal{F}_{c}(0)=f_{\text {Edd }}$ is constant. We then have, from equation 2 ,

$$
\int_{0}^{\infty} \frac{d p_{c}}{d \xi} d \xi=-f_{\mathrm{Edd}} \int_{0}^{\infty} r^{q} d \xi=-f_{\mathrm{Edd}} \frac{r(0)^{q+1}}{q+1},
$$

where $q$ is the index describing the scaling of the diffusion coefficient with the ambient density and $r(0)$ is the value of $r$ at $\xi=0$, and we have taken $r \rightarrow 0$ as $\xi \rightarrow \infty$. The quantity $r(0)^{q+1} /(q+1)$ is of order unity, and thus we recover, in dimensional terms

$$
\dot{\Pi}_{c} \sim P_{*} f_{\text {Edd }} .
$$

If we now rewrite $f_{\text {Edd }}$ in terms of the injected CR flux $F_{c, 0}$ using equation 17 and equation 11, and dropping factors of order unity, we arrive at

$$
\dot{\Pi}_{c} \sim F_{c, 0} \frac{z_{*}}{\kappa_{*}} .
$$

The quantity $\kappa_{*} / z_{*}$ has units of velocity, and can be thought of as the effective velocity which which CRs diffuse, which is lower than the true microphysical velocity by a factor of $\tau_{\mathrm{CR}}$. Thus if we further assume that CRs have a microphysical speed of $c$ in between scatterings, then it immediately follows that

$$
\dot{\Pi}_{c} \sim \tau_{\mathrm{CR}} \frac{F_{c, 0}}{c},
$$

which is exactly the Socrates et al. result.

With this understood, we can now explain why Socrates et al.'s results differ from our equation 51. In the absence of losses, the CR pressure that can build up inside the disc is limited only by considerations of hydrostatic equilibrium. If one considers only CR transfer, then for a sufficiently small value of the CR diffusion coefficient $\kappa_{*}$ (or its dimensionless analog $K_{*}$ ), the Eddington ratio $f_{\text {Edd }}$ can become arbitrarily large, allowing $\dot{\Pi}_{c}$ to become similarly large. However, it is not self-consistent to retain the assumptions that $\tau_{\text {stream }} \sim 0$ and $\tau_{\text {abs }} \sim 0$ as $K_{*} \rightarrow 0$ - from equation 9 and equation 10 , we see that $\tau_{\text {stream }}$ and $\tau_{\text {abs }}$ both scale as $1 / K_{*}$. Thus if a galactic disc has small $K_{*}$, possibly allowing a large CR pressure to build up, it necessarily also has large $\tau_{\text {stream }}$ and $\tau_{\text {abs }}$, which reduce or counteract that buildup. Mathematically, this effect manifests in the fact that equation 51 has a coefficient of $\left[1-f_{\text {cal }}-\mathcal{F}_{c}(\infty) / f_{\text {Edd }}\right] / \beta_{S}$, which approaches Socrates et al.'s factor $\tau_{\mathrm{CR}}$ as $\tau_{\mathrm{abs}} \rightarrow 0$ and $\tau_{\text {stream }} \rightarrow 0$, but is smaller outside of these limits. Physically, the effect is that, if one attempts to confine CRs by making their diffusion slow, then at the same time this raises the importance of streaming and hadronic losses, which set limits on the extent to which the CR pressure can build up.

This paper has been typeset from a $\mathrm{T}_{\mathrm{E}} \mathrm{X} / \mathrm{LAT}_{\mathrm{E}} \mathrm{X}$ file prepared by the author.

9 Note that Socrates et al. do consider CR losses elsewhere in their manuscript (see, for instance, their Appendix C). 